\title{
Synthesis and Antifungal Activity of Aromatic Bis- $\gamma$-lactones Analogous to Avenaciolide
}

\author{
by Pedro A. Castelo-Branco $\left.{ }^{\mathrm{a}}\right)^{\mathrm{b}}$ ), Mayura M. M. Rubinger*a), Leandro de C. Alves ${ }^{\mathrm{a}}$ ), \\ Pauline M. de Barros ${ }^{a}$ ), Sinval G. Pereira ${ }^{a}$ ), Vanessa J. de Melo ${ }^{a}$ ), Dorila Pilo-Veloso ${ }^{b}$ ), \\ and Laércio Zambolim ${ }^{\mathrm{c}}$ )
}

a) Departamento de Química, CCE, Universidade Federal de Viçosa, 36570-000, Viçosa - MG, Brazil (fax: 55-31-38993065; e-mail: mayura@ufv.br)

b) Departamento de Química, ICEx, Universidade Federal de Minas Gerais, 31270-901, Belo Horizonte - MG, Brazil

c) Departamento de Fitopatologia, CCA, Universidade Federal de Viçosa, 36570-000, Viçosa - MG, Brazil

Avenaciolide is a bis- $\gamma$-lactone isolated from Aspergillus avenaceus and possesses antifungal activity. Here, we describe the total syntheses and characterization by elemental analyses, and IR and NMR spectroscopy of three new bis- $\gamma$-lactones analogous to avenaciolide, where the octyl group of the natural product was replaced by aromatic groups. The effects of the avenaciolide, the novel compounds, and their synthetic precursors on the mycelia development and conidia germination of Colletotrichum gloeosporioides were evaluated in vitro. The new compounds were as active as avenaciolide in the tested conditions, while the synthetic precursors were inactive. The preparation and characterization of 15 new synthetic intermediates are also described.

Introduction. - The control of fungal diseases on plants often requires the use of fungicides, and there is a continuous need for new classes of antifungal agents due to the development of resistant strains. Avenaciolide (Fig.) is a naturally occurring antifungal bis- $\gamma$-lactone isolated from Aspergillus avenaceus [1]. Its structure was established by total synthesis and crystallographic studies [2][3]. Avenaciolide has also antibacterial action [1], inhibits the transport of glutamate in rat liver mitochondria [4], and interferes with the ability of ADP to stimulate the rate of glutamate oxidation [5]. Due to all these important biological activities combined with an interesting bicyclic structure, several synthetic approaches to avenaciolide have been published [6][7].<smiles>C=C1C(=O)O[C@H]2C(=O)O[C@@H](CCCCCCCC)[C@H]12</smiles>

Figure. Chemical structure of avenaciolide

We have previously described the preparation of three aliphatic avenaciolide analogs [8], which were active against Colletotrichum, an important fungal genus that causes the plant disease known as anthracnose. Colletotrichum has a wide host range 
including vegetables, field and forage crops, fruit trees, and ornamentals. Here, we describe the synthesis, characterization, and antifungal-activity evaluation against Colletotrichum gloeosporioides of three new avenaciolide analogs containing aromatic substituents. A large number of natural products containing an $\alpha$-methylidene- $\gamma$ lactone group show a wide range of biological activities [9]. To investigate the importance of the $\mathrm{O}=\mathrm{CC}=\mathrm{CH}_{2}$ system for the antifungal activity of avenaciolide and related compounds, the saturated synthetic precursors were also tested.

Results and Discussion. - Syntheses of Bis- $\gamma$-lactones. As the use of a carbohydrate as starting material [2] would enable us to prepare enantiomerically pure avenaciolide analogs, the synthetic approach shown in the Scheme was used for the preparation of compounds 7a-7c, which bear aromatic groups in the side chain. These compounds and all the new synthetic intermediates (i.e., compounds 3-6; Scheme) were characterized by elemental analyses, and IR and NMR spectroscopy. The analyses of the NMR spectra were supported by DEPT, COSY, HMBC, and HSQC experiments.

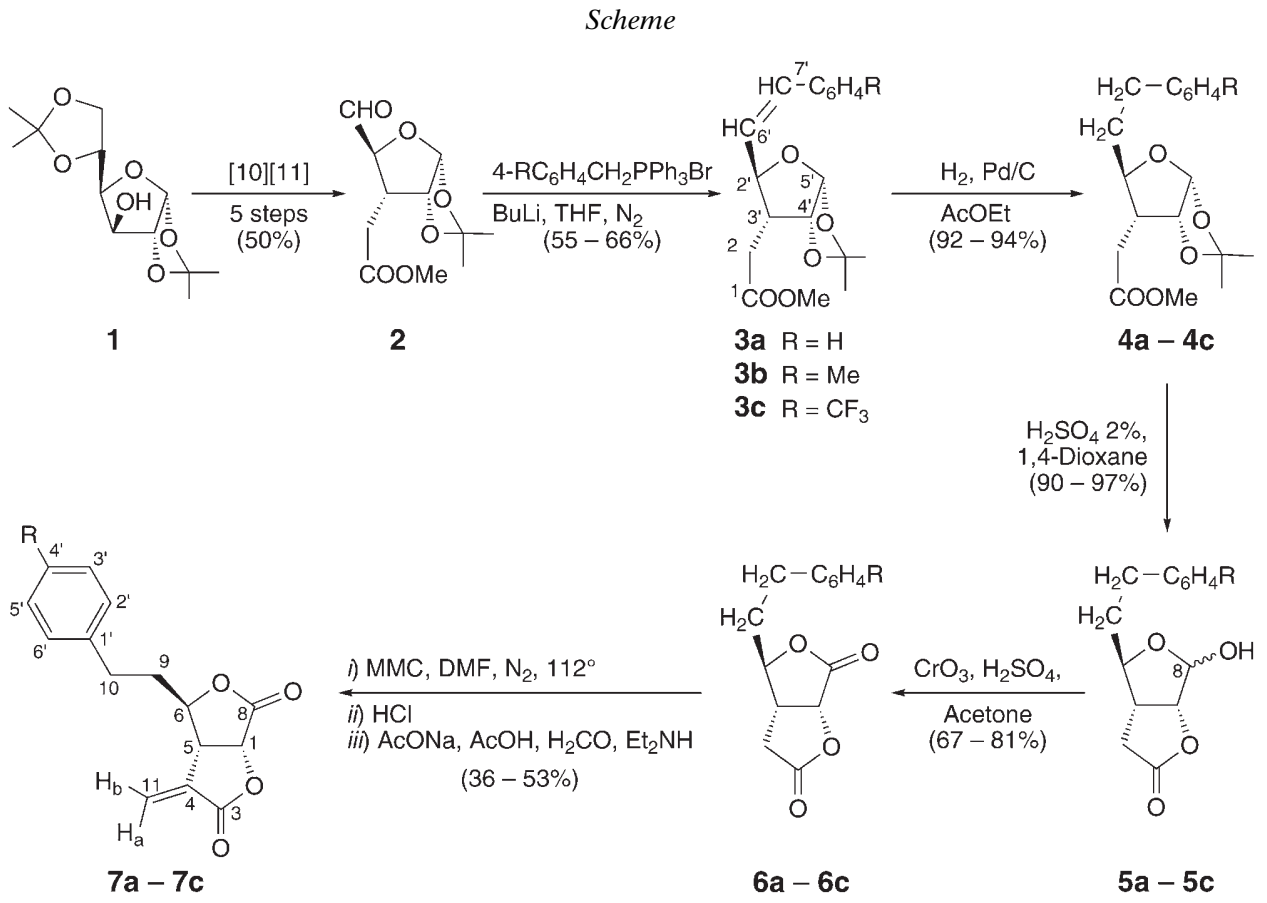

The aldehyde intermediate $\mathbf{2}$ was prepared from diacetone-D-glucose $\mathbf{1}$ as described in [10][11]. The different side chains were introduced by the Wittig reaction of the aldehyde $\mathbf{2}$ and the phosphonium ylides prepared in situ from the Wittig salts and BuLi, to yielding mixtures of $(Z / E)$-isomers $\mathbf{3 a}-\mathbf{3 c}$. The Scheme displays the arbitrary numbering of the $\mathrm{C}$-atoms used for the NMR-signals attributions. The signals of the olefinic $\mathrm{C}\left(6^{\prime}\right)$ and $\mathrm{C}\left(7^{\prime}\right)$ of the major $(Z)$-isomers were observed at $c a .127$ and $136 \mathrm{ppm}$, respectively, in the ${ }^{13} \mathrm{C}-\mathrm{NMR}$ spectra of compounds 3a-3c. Along with these signals, 
other less intense ones were indicative of the presence of the $(E)$-isomers as minor components. The coupling constant $(J)$ of $c a$. $11 \mathrm{~Hz}$ for the olefinic $\mathrm{H}$-atoms $\mathrm{H}-\mathrm{C}\left(6^{\prime}\right)$ and $\mathrm{H}-\mathrm{C}\left(7^{\prime}\right)$ observed in the ${ }^{1} \mathrm{H}-\mathrm{NMR}$ spectra of these compounds confirmed the $(Z)$ configuration for the main component. The observation of the expected signals in the aromatic range $(7.1-7.6 \mathrm{ppm})$ confirmed the presence of the aromatic groups in the structures of $\mathbf{3 a}-\mathbf{3 c}$. It was not necessary to separate the $(Z / E)$-isomers to follow the synthetic approach chosen.

The hydrogenation of $\mathbf{3 a}-\mathbf{3 c}$ yielded the compounds $\mathbf{4 a}-\mathbf{4 c}$, respectively, which, in the ${ }^{13} \mathrm{C}$-NMR spectra, showed the two $\mathrm{CH}_{2}$ signals at $c a .34$ and $32 \mathrm{ppm}$, attributed to $\mathrm{C}\left(6^{\prime}\right)$ and $\mathrm{C}\left(7^{\prime}\right)$ in accordance with the correlations observed in their HSQC contour maps $\left(\mathrm{H}-\mathrm{C}\left(6^{\prime}\right)\right.$ at $1.6-1.9 \mathrm{ppm}$ and $\mathrm{H}-\mathrm{C}\left(7^{\prime}\right)$ at 2.6-2.9 ppm $)$. Further, the correlation $\left({ }^{3} J\right)$ observed between the aromatic $\mathrm{C}\left(2^{\prime \prime}\right)$ and $\mathrm{C}\left(6^{\prime \prime}\right)$ with the $\mathrm{H}-\mathrm{C}\left(7^{\prime}\right)$ signals in the HMBC contour map confirmed such attributions. The HMBC also showed a correlation $\left({ }^{2} J\right)$ of $\mathrm{H}-\mathrm{C}\left(7^{\prime}\right)$ signals to $\mathrm{C}\left(1^{\prime \prime}\right)$, while the $\mathrm{H}-\mathrm{C}\left(6^{\prime}\right)$ signals were correlated only to this aromatic ${ }^{13} \mathrm{C}$ signal $\left({ }^{3} J\left(\mathrm{H}-\mathrm{C}\left(6^{\prime}\right), \mathrm{C}\left(1^{\prime \prime}\right)\right)\right.$.

The first lactone ring was closed by the reaction of the esters $4 \mathbf{a}-\mathbf{4 c}$ with $\mathrm{H}_{2} \mathrm{SO}_{4}$ $(2 \%)$ in 1,4-dioxane under reflux (Scheme). The several duplicated characteristic signal groups in the NMR spectra of $\mathbf{5 a - 5 c}$ indicated the formation of mixtures of $\mathrm{C}(8)$ epimers. For example, two broad signals were observed for the $\mathrm{OH}$ groups (at $c a .3 .7$ and $3.4 \mathrm{ppm}$ for $\mathrm{OH}$ in $\alpha$ - and $\beta$-positions, at a $1: 2$ proportion, resp.) in the ${ }^{1} \mathrm{H}-\mathrm{NMR}$ spectrum of $\mathbf{5 a}$. These signals were obscured by other signals in the spectra of $\mathbf{5 b}$ and $\mathbf{5 c}$, but the integration curves were indicative of their presence. The identity of the major product was established by the comparison of the $\mathrm{H}-\mathrm{C}(8)$ signals, which appeared as singlets at ca. $5.6 \mathrm{ppm}$ for the epimers with the $\mathrm{OH}$ group in $\beta$-position, and as doublets $(J(8,1) \approx 4 \mathrm{~Hz})$ at $5.5 \mathrm{ppm}$ for the minor $\alpha$-isomers. The correlated $\mathrm{C}(8)$ signals were observed at $c a$. 101 and $96 \mathrm{ppm}$ (less intense), respectively, in the ${ }^{13} \mathrm{C}$-NMR spectra of the mixtures 5a-5c. The $\mathrm{C}=\mathrm{O}$ stretching band was observed in the IR spectra of $5 \mathbf{a}$ and $\mathbf{5 b}$ as a broad band centered at 1781 and $1755 \mathrm{~cm}^{-1}$, respectively. Interestingly, two distinct bands were observed in the spectrum of $\mathbf{5 c}$ at 1780 and $1746 \mathrm{~cm}^{-1}$ (less intense). The most intense band was attributed to the major product, with the $\mathrm{OH}$ group in $\beta$-position. The lower wave number observed for the $\alpha$-isomer is probably due to intramolecular $\mathrm{H}$-bonding with the $\mathrm{O}$-atom at $\mathrm{C}(3)$.

The Jones oxidation of the compounds $5 \mathbf{a}-\mathbf{5 c}$ yielded the bis- $\gamma$-lactones $\mathbf{6 a}-\mathbf{6 c}$, respectively. In the IR spectra of $\mathbf{6 a}$ and $\mathbf{6 b}$, two distinct $\tilde{v}(\mathrm{C}=\mathrm{O})$ absorptions could be observed (at $c a .1775$ and $1795 \mathrm{~cm}^{-1}$ ), while the spectrum of $\mathbf{6 c}$ showed a broad band centered at $1779 \mathrm{~cm}^{-1}$. The simplification of the $\mathrm{H}-\mathrm{C}(1)$ signal multiplicity in the ${ }^{1} \mathrm{H}-\mathrm{NMR}$ spectra of these compounds (doublets at ca. $5.01 \mathrm{ppm}$, with $J(1,5) \approx 8 \mathrm{~Hz}$ ) and the observation of the two $\mathrm{C}=\mathrm{O}$ signals in their ${ }^{13} \mathrm{C}$-NMR spectra $(\mathrm{C}(8)$ at $c a .170$ and $\mathrm{C}(3)$ at $c a .173 \mathrm{ppm}$ ) confirmed the oxidation at $\mathrm{C}(8)$. The attributions of the $\mathrm{C}=\mathrm{O}$ signals were supported by the HMBC contour maps which showed ${ }^{3} J$ correlations between $\mathrm{C}(8)$ and $\mathrm{H}-\mathrm{C}(6)$ signals, and ${ }^{2} J$ correlations between $\mathrm{C}(3)$ and $\mathrm{H}_{\mathrm{a}}-\mathrm{C}(4)$ and $\mathrm{H}_{\mathrm{b}}-\mathrm{C}(4)$ signals.

The exocyclic methylene group was introduced into the bis- $\gamma$-lactone skeleton using the methodology described by Parker and Johnson [12]. The reaction of $\mathbf{6 a - 6 \mathbf { c }}$ with magnesium methyl carbonate (MMC; Scheme), followed by the addition of $\mathrm{HCl}$, yielded the carboxylic acid intermediates, which were not isolated. To the crude 
products (yellow oils) was added a mixture of $\mathrm{AcONa}, \mathrm{AcOH}, \mathrm{CH}_{2} \mathrm{O}, \mathrm{H}_{2} \mathrm{O}$, and $\mathrm{Et}_{2} \mathrm{NH}$, to yield the avenaciolide analogs $7 \mathbf{a}-7 \mathbf{c}$. The IR spectra of $\mathbf{7 a}-\mathbf{7 c}$ showed additional bands due to the additional $\mathrm{C}=\mathrm{C}$ stretching vibration (at $c a .1665 \mathrm{~cm}^{-1}$ ). The spectra of compounds 7a and 7c showed a broad band in the $\mathrm{C}=\mathrm{O}$ region of the $\gamma$-lactones, and, in the spectrum of $\mathbf{7 b}$, two well-resolved bands were observed at 1791 and $1760 \mathrm{~cm}^{-1}$, which can be attributed to $\mathrm{C}(8)=\mathrm{O}$ and $\mathrm{C}(3)=\mathrm{O}$, respectively. No changes were observed in the $\mathrm{C}(8)=\mathrm{O}$ signals in the ${ }^{13} \mathrm{C}$-NMR spectra of compounds $7 \mathbf{a}-7 \mathbf{c}$, when compared to the spectra of the parent bis-lactones. On the other hand, the introduction of the methylidene group $\alpha$ to $\mathrm{C}(3)$ caused the expected shift on this $\mathrm{C}=\mathrm{O}$ signal from ca. 173 to $167 \mathrm{ppm}$. The HMBC contour maps for $7 \mathbf{a}-7 \mathbf{c}$ showed ${ }^{3} J$ correlations between $\mathrm{C}(3)$ and $\mathrm{H}_{\mathrm{a}}-\mathrm{C}(11)$ and $\mathrm{H}_{\mathrm{b}}-\mathrm{C}(11)$ signals, and ${ }^{4} J$ correlations between $\mathrm{C}(8)$ and $\mathrm{H}_{\mathrm{a}}-\mathrm{C}(9)$ and $\mathrm{H}_{b}-\mathrm{C}(9)$ signals, confirming the $\mathrm{C}=\mathrm{O}$ signal attributions. The expected change in the chemical shifts of $\mathrm{C}(4)$ signals from $c a .33 \mathrm{ppm}$ in the ${ }^{13} \mathrm{C}-\mathrm{NMR}$ spectra of the parent bis- $\gamma$-lactones to $c a$. $134 \mathrm{ppm}$ confirmed the formation of compounds 7a-7c. As in the ${ }^{1} \mathrm{H}-\mathrm{NMR}$ spectrum of avenaciolide [13], the signals of the vinylic $\mathrm{H}_{\mathrm{a}}-\mathrm{C}(11)$ and $\mathrm{H}_{\mathrm{b}}-\mathrm{C}(11)$ of the analogs $7 \mathbf{a}-\mathbf{7 c}$ appeared as two doublets at $c a$. 6.4 and $5.8 \mathrm{ppm}$ in their spectra. The correlated C-signals (HSQC) were observed at $c a$. $126.5 \mathrm{ppm}$ in the ${ }^{13} \mathrm{C}-\mathrm{NMR}$ spectra of $\mathbf{7 a - 7 c}$. The COSY contour maps showed a ${ }^{4} \boldsymbol{J}$ coupling between the $\mathrm{H}-\mathrm{C}(11)$ signals and $\mathrm{H}-\mathrm{C}(5)$, which is also coupled to $\mathrm{H}-\mathrm{C}(1)$ and $\mathrm{H}-\mathrm{C}(6)$. The remaining signals were in very good agreement with the proposed structures.

Antifungal Screening. The bis- $\gamma$-lactones $6 \mathbf{a}-\mathbf{6 c}$ and $7 \mathbf{a}-7 \mathbf{c}$, and avenaciolide were tested for their capacity to inhibit the growth of the phytopathogenic fungi Colletotrichum gloeosporioides. The test methodology was designed in order to allow the use of very low amounts of substances and to provide a fast way to evaluate the antifungal potential of the compounds. The commercial fungicide Folicur of the triazole group of fungicides used as a positive control is employed on the control of $C$. gloeosporioides in several cultures such as citrus, mango, papaya, guava, passion fruit, and grapes. It contains tebuconazole $\left(200 \mathrm{~g} \mathrm{l}^{-1}\right)$ as active ingredient and, under the test conditions, gives good responses at the very low dose employed (inhibition hale of $c a$. $4 \mathrm{~cm}$ in $48 \mathrm{~h}$ ). Paper discs $(6 \mathrm{~mm})$ were dipped into the solutions of the compounds at $1000 \mathrm{ppm}$. The discs were removed from the solutions and, after evaporation of the solvent, they were placed in the center of Petri dishes containing C. gloeosporioides conidia mixed with the BDA media. After $48 \mathrm{~h}$ of incubation at $25^{\circ}$, compounds $7 \mathbf{a}-7 \mathbf{c}$ and avenaciolide presented inhibition hales (limited by the region where a dense and filamentous growth could be observed with the aid of a stereoscopic microscope) of 39$47 \%$ of the diameter of the positive control (Folicur). It has been shown that other $\alpha-$ methylidene- $\gamma$-lactones react rapidly with enzymes to form stable adducts, explaining at least in part their biological activity [14][15]. Compounds $\mathbf{6 a - 6 \mathbf { c }}$ were totally inactive under the test conditions. These results indicate that not only the bis- $\gamma$-lactone skeleton is important for the antifungal activity of these compounds, but also it depends on the presence of the exocyclic $\mathrm{C}=\mathrm{C}$ bond probably due to a Michael-type addition reaction with the fungi enzymes.

The small differences on the aromatic groups of compounds $7 \mathbf{a}-\mathbf{7 c}$ did not lead to great differences in their antifungal activities in the in vitro test employed. In fact, the results for $\mathbf{7 b}$ and $\mathbf{7 c}(1.5 \mathrm{~cm}, 39 \%$ inhibition $)$ could not be differentiated by using the 
Scott-Knott test at $5 \%$ of probability [16]. By a similar methodology, but at the concentration of $100 \mathrm{ppm}$ and with a different positive control (benomyl), it was observed for aliphatic analogs containing pentyl, hexyl, and heptyl groups [8] that the increase in the C-chain length seems to increase the activity (39, 40, and 46\%, resp.). The same feature was not observed in these tests, and compound 7a was found more active than $\mathbf{7 b}$ and $\mathbf{7 c}$. The activity of $\mathbf{7 a}(47 \%)$ was in the same range of that presented by avenaciolide $(45 \%)$, showing that the increase of the C-chain length is not the only factor influencing the antifungal activity of these bis-lactones. It is noteworthy that another very interesting result could be observed in these tests: although the tebuconazole causes a much broader inhibition hale, the Petri dishes containing the compounds $7 \mathbf{a}-\mathbf{7 c}$ or avenaciolide showed a completely transparent hale, while the hale of the control was homogeneously opaque.

To further investigate the biological activities of this class of compounds and to evaluate their applicability as agrochemicals, other analogues are being prepared, and tests of different methodologies will be carried out.

We thank the Brazilian agencies FAPEMIG (CEX-860/02) for financial support, and $C N P q$ and CAPES for students' grants.

\section{Experimental Part}

General. Diacetone-D-glucose $\mathbf{1}$ and the aryl bromides $\left(\mathrm{PhCH}_{2} \mathrm{Br}\right.$, 4-methylbenzyl bromide, and 4trifluoromethyl bromide) were purchased from Aldrich. Aldehyde $\mathbf{2}$ was prepared from $\mathbf{1}$ as described in [10][11]. Avenaciolide was prepared from $\mathbf{2}$ as described in [2]. Solvents were distilled before use and dried according to standard procedures. Optical rotations: Bellingham + Stanley model $D$ polarimeter. IR Spectra: Perkin Elmer Paragon 1000 spectrometer $\left(4000-400 \mathrm{~cm}^{-1}\right)$ in $\mathrm{KBr}$ (when solids) or as thin films on $\mathrm{NaCl}$ plates (when oils). NMR Spectra: in $\mathrm{CDCl}_{3}$ with a Bruker DRX-400 AVANCE or a Bruker $D P X$-200 AVANCE spectrometer; chemical shifts $\delta$ in ppm rel. to TMS as internal standard, and coupling constants $J$ in $\mathrm{Hz}$; the assignments of signals in NMR spectra of the new compounds $\mathbf{4}-\mathbf{7}$ were supported by 2D experiments (COSY, HSQC, and HMBC contour maps). Microanalyses were performed with a Perkin-Elmer 2400 elemental analyzer.

Preparation of $\mathbf{3 a}-\mathbf{3 c}$. The appropriate aryl bromide $\left(\mathrm{PhCH}_{2} \mathrm{Br}(1.71 \mathrm{~g})\right.$ or 4-methylbenzyl bromide $(1.76 \mathrm{~g})$, or 4-trifluoromethylbenzyl bromide $(2.05 \mathrm{~g}))$ was added to a stirring soln. of $\mathrm{Ph}_{3} \mathrm{P}(2.62 \mathrm{~g}, 3.55 \mathrm{~g}$ and $3.20 \mathrm{~g}$, resp.) in dry benzene $(4 \mathrm{ml})$ at r.t. under $\mathrm{N}_{2}$. The mixture was stirred under reflux for $2 \mathrm{~h}$. The product was filtered, washed with $\mathrm{Et}_{2} \mathrm{O}$ and dried under reduced pressure yielding the Wittig salts as white solids ((benzyl)(triphenyl)phosphonium bromide (a, $4.20 \mathrm{~g}, 97 \%)$, (4-methylbenzyl)(triphenyl)phosphonium bromide (b, $3.83 \mathrm{~g}, 90 \%)$ and [4-(trifluoromethyl)benzyl](triphenyl)phosphonium bromide (c, $4.08 \mathrm{~g}, 95 \%))$. BuLi (15\% in hexane; $2.8,2.7$, or $2.6 \mathrm{ml}$ for $\mathbf{a}, \mathbf{b}$, or $\mathbf{c}$, resp.) was added to a stirring soln. of the Wittig salt (a: $2.64 \mathrm{~g}, 6.1 \mathrm{mmol} ; \mathbf{b}: 3.21 \mathrm{~g}, 6.4 \mathrm{mmol}$; or $\mathbf{c}: 2.73 \mathrm{~g}, 6.1 \mathrm{mmol})$ in dry THF (20 ml) under $\mathrm{N}_{2}$. The mixture was stirred for 10 min prior to the addition of a soln. of $2(1.30 \mathrm{~g}, 5.3 \mathrm{mmol})$ in dry THF $(7 \mathrm{ml})$. After $18 \mathrm{~h}$ stirring at r.t., the mixture was concentrated i.v., $\mathrm{H}_{2} \mathrm{O}(25 \mathrm{ml})$ was added, and extractions were performed with $\mathrm{Et}_{2} \mathrm{O}(5 \times 25 \mathrm{ml})$. The org. phase was dried $\left(\mathrm{Na}_{2} \mathrm{SO}_{4}\right)$, concentrated, and submitted to $\mathrm{CC}\left(\mathrm{SiO}_{2}\right.$; hexane/AcOEt $\left.3: 1\right)$ to yield the mixture of isomers $\mathbf{3 a}(0.93 \mathrm{~g}, 55 \%), \mathbf{3 b}$ $0.99 \mathrm{~g}, 56 \%)$, or $3 \mathrm{c}(1.36 \mathrm{~g}, 66 \%)$.

Methyl 2-\{(2R,3R,4R,5R)-2,3,4,5-Tetrahydro-4,5-(isopropylidenedioxy)-2-[(Z)-2-phenylethenyl]furan-3-yl $\}$ acetate and Methyl 2-\{(2R,3R,4R,5R)-2,3,4,5-Tetrahydro-4,5-(isopropylidenedioxy)-2-[(E)2-phenylethenyl]furan-3-yljacetate (3a). White crystals. $R_{\mathrm{f}}$ (hexane/AcOEt $3: 1$ ) 0.51 . M.p. 73.7-76.2 (hexane/AcOEt 3 :1). IR (KBr): 2987, 2942, 1735, 1493, 1437, 1385, 1330, 1209, 1169, 1021, 873, 799, 705. $\left.{ }^{1} \mathrm{H}-\mathrm{NMR}\left(\mathrm{CDCl}_{3}, 400 \mathrm{MHz}\right)^{1}\right): 1.31(s, \mathrm{Me}) ; 1.34(s, \mathrm{Me} *) ; 1.42(s, \mathrm{Me}) ; 1.54\left(s, \mathrm{Me}^{*}\right) ; 2.21-2.27(m$,

1) The signals marked '*' are due to the $(E)$-isomer. All the others refer to the major $(Z)$-isomer. 
$\left.\mathrm{H}_{\mathrm{a}}-\mathrm{C}(2), \mathrm{H}-\mathrm{C}\left(3^{\prime}\right), \mathrm{H}-\mathrm{C}\left(3^{*}\right)\right) ; 2.38\left(d d,{ }^{2} J\left(2 \mathrm{a}^{*}, 2 \mathrm{~b}^{*}\right)=17.4,{ }^{3} J\left(2 \mathrm{a}^{*}, 3^{\prime *}\right)=4.7, \mathrm{H}_{\mathrm{a}}-\mathrm{C}\left(2^{*}\right)\right) ; 2.53(d d$, $\left.{ }^{2} J(2 \mathrm{~b}, 2 \mathrm{a})=17.6,{ }^{3} J\left(2 \mathrm{~b}, 3^{\prime}\right)=11.2, \mathrm{H}_{\mathrm{b}}-\mathrm{C}(2)\right) ; 2.69-2.75\left(m, \mathrm{H}_{\mathrm{b}}-\mathrm{C}\left(2^{*}\right)\right) ; 3.63\left(s, \mathrm{MeO}^{*}\right) ; 3.67(s, \mathrm{MeO})$; $4.31\left(d d,{ }^{3} J\left(2^{\prime *}, 3^{\prime *}\right)=10.7,{ }^{3} J\left(2^{\prime *}, 6^{\prime *}\right)=8.0, \mathrm{H}-\mathrm{C}\left(2^{\prime *}\right)\right) ; 4.63\left(d d,{ }^{3} J\left(2^{\prime}, 3^{\prime}\right)=9.8,{ }^{3} J\left(2^{\prime}, 6^{\prime}\right)=9.8, \mathrm{H}-\mathrm{C}\left(2^{\prime}\right)\right)$; $4.79\left(d d,{ }^{3} J\left(4^{\prime}, 5^{\prime}\right)=3.9,{ }^{3} J\left(4^{\prime}, 3^{\prime}\right)=3.9, \mathrm{H}-\mathrm{C}\left(4^{\prime}\right)\right) ; 4.82\left(d d,{ }^{3} J\left(4^{\prime} *, 5^{\prime *}\right)=4.1,{ }^{3} J\left(4^{\prime *}, 3^{\prime *}\right)=4.1, \mathrm{H}-\mathrm{C}\left(4^{\prime *}\right)\right)$; $5.57\left(d d,{ }^{3} J\left(6^{\prime}, 7^{\prime}\right)=11.4,{ }^{3} J\left(6^{\prime}, 2^{\prime}\right)=9.8, \mathrm{H}-\mathrm{C}\left(6^{\prime}\right)\right) ; 5.90\left(d,{ }^{3} J\left(5^{\prime}, 4^{\prime}\right)=3.9, \mathrm{H}-\mathrm{C}\left(5^{\prime}\right)\right) ; 6.07\left(d d,{ }^{3} J\left(6^{\prime *}, 7^{\prime *}\right)=\right.$ $\left.15.8,{ }^{3} J\left(6^{\prime *}, 2^{\prime *}\right)=8.4, \mathrm{H}-\mathrm{C}\left(6^{\prime *}\right)\right) ; 6.65\left(d,{ }^{3} J\left(7^{\prime *}, 6^{\prime *}\right)=15.6, \mathrm{H}-\mathrm{C}\left(7^{* *}\right)\right) ; 6.81\left(d,{ }^{3} J\left(7^{\prime}, 6^{\prime}\right)=11.4\right.$, $\left.\mathrm{H}-\mathrm{C}\left(7^{\prime}\right)\right) ; 7.26-7.38\left(m, \mathrm{H}-\mathrm{C}\left(2^{\prime \prime}\right), \mathrm{H}-\mathrm{C}\left(3^{\prime \prime}\right), \mathrm{H}-\mathrm{C}\left(4^{\prime \prime}\right), \mathrm{H}-\mathrm{C}\left(5^{\prime \prime}\right), \mathrm{H}-\mathrm{C}\left(6^{\prime \prime}\right), \mathrm{H}-\mathrm{C}\left(2^{\prime \prime *}\right)\right.$, $\left.\left.\mathrm{H}-\mathrm{C}\left(3^{\prime \prime *}\right), \mathrm{H}-\mathrm{C}\left(4^{\prime \prime *}\right), \mathrm{H}-\mathrm{C}\left(5^{\prime \prime *}\right), \mathrm{H}-\mathrm{C}\left(6^{\prime \prime *}\right)\right) .{ }^{13} \mathrm{C}-\mathrm{NMR}\left(\mathrm{CDCl}_{3}, 100 \mathrm{MHz}\right)^{1}\right): 26.3(2 \mathrm{Me} *) ; 26.5$ (2 Me); $29.1(\mathrm{C}(2)) ; 29.4\left(\mathrm{C}\left(2^{*}\right)\right) ; 45.9\left(\mathrm{C}\left(3^{\prime *}\right)\right) ; 46.6\left(\mathrm{C}\left(3^{\prime}\right)\right) ; 51.7\left(\mathrm{MeO}^{*}\right) ; 51.8(\mathrm{MeO}) ; 76.0\left(\mathrm{C}\left(2^{\prime}\right)\right)$; $80.8\left(\mathrm{C}\left(4^{\prime}\right)\right) ; 81.9\left(\mathrm{C}\left(2^{\prime *}\right)\right) ; 105.0\left(\mathrm{C}\left(5^{\prime}\right)\right) ; 111.6\left(\mathrm{Me}_{2} \mathrm{C}\right) ; 111.7\left(\mathrm{Me}_{2} \mathrm{C}^{*}\right) ; 126.1\left(\mathrm{C}\left(6^{\prime *}\right)\right) ; 126.7\left(\mathrm{C}\left(2^{\prime \prime *}\right)\right.$, $\left.\mathrm{C}\left(6^{\prime \prime *}\right)\right) ; 127.7\left(\mathrm{C}\left(6^{\prime}\right), \mathrm{C}\left(4^{\prime \prime}\right)\right) ; 128.1\left(\mathrm{C}\left(4^{\prime \prime *}\right)\right) ; 128.3\left(\mathrm{C}\left(3^{\prime \prime}\right), \mathrm{C}\left(5^{\prime \prime}\right)\right) ; 128.6\left(\mathrm{C}\left(3^{\prime \prime *}\right), \mathrm{C}\left(5^{\prime \prime *}\right)\right) ; 128.8\left(\mathrm{C}\left(2^{\prime \prime}\right)\right.$, $\left.\mathrm{C}\left(6^{\prime \prime}\right)\right) ; 134.5\left(\mathrm{C}\left(7^{\prime *}\right)\right) ; 136.0\left(\mathrm{C}\left(1^{\prime \prime}\right)\right) ; 136.1\left(\mathrm{C}\left(1^{\prime \prime *}\right)\right) ; 136.8\left(\mathrm{C}\left(7^{\prime}\right)\right) ; 172.0\left(\mathrm{C}\left(1^{*}\right)\right) ; 172.5(\mathrm{C}(1))$. Anal. calc. for $\mathrm{C}_{18} \mathrm{H}_{22} \mathrm{O}_{5}$ (318.37): C 67.91, H 6.97; found: C 68.14, H 7.01.

Methyl 2-\{(2R,3R,4R,5R)-2,3,4,5-Tetrahydro-4,5-(isopropylidenedioxy)-2-[(Z)-2-(4-methylphenyl)ethenyl]furan-3-yl $\}$ acetate and Methyl 2- $\{(2 \mathrm{R}, 3 \mathrm{R}, 4 \mathrm{R}, 5 \mathrm{R})-4,5$-isopropylidenedioxy-[(E)-2-(4-methylphenyl)ethenylffuran-3-yljacetate (3b). White crystals. $R_{\mathrm{f}}$ (hexane/AcOEt $3: 1$ ) 0.54 . M.p. 109.5-112.5 (hexane/AcOEt 3 :1). IR (KBr): 2989, 2957, 1735, 1511, 1435, 1373, 1328, 1209, 1169, 1023, 965, 872, 835. $\left.{ }^{1} \mathrm{H}-\mathrm{NMR}\left(\mathrm{CDCl}_{3}, 200 \mathrm{MHz}\right)^{1}\right): 1.32(s, \mathrm{Me}) ; 1.34(s, \mathrm{Me} *) ; 1.45(s, \mathrm{Me}) ; 1.54(s, \mathrm{Me} *) ; 2.10-2.35(\mathrm{~m}$, $\left.\mathrm{H}_{\mathrm{a}}-\mathrm{C}(2), \mathrm{H}-\mathrm{C}\left(3^{\prime}\right), \mathrm{H}_{\mathrm{a}}-\mathrm{C}\left(2^{*}\right), \mathrm{H}-\mathrm{C}\left(3^{\prime *}\right)\right) ; 2.35\left(s, \mathrm{Me}-\mathrm{C}\left(4^{\prime \prime}\right)\right) ; 2.54\left(d d,{ }^{2} \mathrm{~J}(2 \mathrm{~b}, 2 \mathrm{a})=17.4,{ }^{3} J\left(2 \mathrm{~b}, 3^{\prime}\right)=\right.$ $\left.11.1, \mathrm{H}_{\mathrm{b}}-\mathrm{C}(2)\right) ; 2.68\left(d d,{ }^{2} J\left(2 \mathrm{~b}^{*}, 2 \mathrm{a}^{*}\right)=16.5,{ }^{3} J\left(2 \mathrm{~b}^{*}, 3^{\prime *}\right)=9.9, \mathrm{H}_{\mathrm{b}}-\mathrm{C}(2 *)\right) ; 3.63(s, \mathrm{MeO} *) ; 3.67(s, \mathrm{MeO})$; $4.25-4.34\left(m, \mathrm{H}-\mathrm{C}\left(2^{\prime *}\right)\right) ; 4.65\left(d d,{ }^{3} J\left(2^{\prime}, 3^{\prime}\right)=9.7,{ }^{3} J\left(2^{\prime}, 6^{\prime}\right)=9.7, \mathrm{H}-\mathrm{C}\left(2^{\prime}\right)\right) ; 4.77-4.84\left(m, \mathrm{H}-\mathrm{C}\left(4^{\prime *}\right)\right)$; $4.79\left(d d,{ }^{3} J\left(4^{\prime}, 5^{\prime}\right)=3.8,{ }^{3} J\left(4^{\prime}, 3^{\prime}\right)=3.8, \mathrm{H}-\mathrm{C}\left(4^{\prime}\right)\right) ; 5.51\left(d d,{ }^{3} J\left(6^{\prime}, 7^{\prime}\right)=11.2,{ }^{3} J\left(6^{\prime}, 2^{\prime}\right)=9.6, \mathrm{H}-\mathrm{C}\left(6^{\prime}\right)\right) ; 5.90(d$, $\left.{ }^{3} J\left(5^{\prime}, 4^{\prime}\right)=3.8, \mathrm{H}-\mathrm{C}\left(5^{\prime}\right)\right) ; 6.00\left(d d,{ }^{3} J\left(6^{\prime *}, 7^{*} *\right)=15.9,{ }^{3} J\left(6^{\prime *}, 2^{\prime *}\right)=8.2, \mathrm{H}-\mathrm{C}\left(6^{\prime *}\right)\right) ; 6.62\left(d,{ }^{3} J\left(7^{\prime *}, 6^{\prime *}\right)=\right.$ $\left.15.9, \mathrm{H}-\mathrm{C}\left(7^{\prime *}\right)\right) ; 6.77\left(d,{ }^{3} J\left(7^{\prime}, 6^{\prime}\right)=11.2, \mathrm{H}-\mathrm{C}\left(7^{\prime}\right)\right) ; 7.10-7.17\left(m, \mathrm{H}-\mathrm{C}\left(3^{\prime \prime}\right), \mathrm{H}-\mathrm{C}\left(5^{\prime \prime}\right), \mathrm{H}-\mathrm{C}\left(3^{\prime \prime *}\right)\right.$, $\left.\left.\mathrm{H}-\mathrm{C}\left(5^{\prime \prime *}\right)\right) ; 7.25-7.29\left(m, \mathrm{H}-\mathrm{C}\left(2^{\prime \prime}\right), \mathrm{H}-\mathrm{C}\left(6^{\prime \prime}\right), \mathrm{H}-\mathrm{C}\left(2^{\prime \prime *}\right), \mathrm{H}-\mathrm{C}\left(6^{\prime \prime *}\right)\right) \cdot{ }^{13} \mathrm{C}-\mathrm{NMR}\left(\mathrm{CDCl}_{3}, 50 \mathrm{MHz}\right)^{1}\right)$ : $21.2\left(\mathrm{Me}-\mathrm{C}\left(4^{\prime \prime}\right)\right) ; 26.3\left(\mathrm{Me}^{*}\right) ; 26.5(\mathrm{Me}) ; 26.6\left(\mathrm{Me}, \mathrm{Me}^{*}\right) ; 29.1\left(\mathrm{C}(2), \mathrm{C}\left(2^{*}\right)\right) ; 45.9\left(\mathrm{C}\left(3^{\prime *}\right)\right) ; 46.6\left(\mathrm{C}\left(3^{\prime}\right)\right)$; $51.7(\mathrm{MeO}, \mathrm{MeO} *) ; 76.1\left(\mathrm{C}\left(2^{\prime}\right)\right) ; 80.7\left(\mathrm{C}\left(4^{\prime *}\right)\right) ; 80.8\left(\mathrm{C}\left(4^{\prime}\right)\right)$; $82.1\left(\mathrm{C}\left(2^{\prime *}\right)\right) ; 105.0\left(\mathrm{C}\left(5^{\prime}\right), \mathrm{C}\left(5^{\prime *}\right)\right) ; 111.6$ $\left(\mathrm{Me}_{2} \mathrm{C}\right) ; 111.7\left(\mathrm{Me}_{2} \mathrm{C}^{*}\right) ; 124.9\left(\mathrm{C}\left(6^{\prime *}\right)\right) ; 126.6\left(\mathrm{C}\left(2^{\prime \prime *}\right), \mathrm{C}\left(6^{\prime \prime *}\right)\right) ; 126.9\left(\mathrm{C}\left(6^{\prime}\right)\right) ; 128.8\left(\mathrm{C}\left(2^{\prime \prime}\right), \mathrm{C}\left(6^{\prime \prime}\right)\right) ; 129.0$ $\left(\mathrm{C}\left(3^{\prime \prime}\right), \mathrm{C}\left(5^{\prime \prime}\right)\right) ; 129.3\left(\mathrm{C}\left(3^{\prime \prime *}\right), \mathrm{C}\left(5^{\prime \prime *}\right)\right) ; 133.1\left(\mathrm{C}\left(1^{\prime \prime}\right)\right) ; 133.3\left(\mathrm{C}\left(1^{\prime \prime *}\right)\right) ; 134.5\left(\mathrm{C}\left(7^{\prime *}\right)\right) ; 136.8\left(\mathrm{C}\left(7^{\prime}\right)\right) ; 137.6$ $\left(\mathrm{C}\left(4^{\prime \prime}\right)\right) ; 138.1\left(\mathrm{C}\left(4^{\prime \prime *}\right)\right) ; 171.2\left(\mathrm{C}\left(1^{*}\right)\right) ; 172.5(\mathrm{C}(1))$. Anal. calc. for $\mathrm{C}_{19} \mathrm{H}_{24} \mathrm{O}_{5}(332.40): \mathrm{C} 68.66, \mathrm{H}$ 7.28; found: C 69.13, H 6.84 .

Methyl 2-((2R,3R,4R,5R)-2,3,4,5-Tetrahydro-4',5'-(isopropylidenedioxy)-2-\{(Z)-2-[4-(trifluoromethyl)phenyl]ethenylffuran-3-yl)acetate and Methyl 2-(2R,3R,4R,5R)-2,2,4,5-Tetrahydro-4,5-(isopropylidenedioxy)-2-\{(E)-2-(4-[4-(trifluoromethyl)phenyl]ethenyl\}furan-3-yl)acetate $(3 \mathrm{c})$. Colorless oil. $R_{\mathrm{f}}$ (hexane/AcOEt 3 :1) 0.46. IR (film): 2989, 2955, 1739, 1617, 1439, 1383, 1375, 1326, 1217, 1166, 1127, 1067, 1017, 864. $\left.{ }^{1} \mathrm{H}-\mathrm{NMR}\left(\mathrm{CDCl}_{3}, 400 \mathrm{MHz}\right)^{1}\right): 1.32(s, \mathrm{Me}) ; 1.35(s, \mathrm{Me} *) ; 1.42(s, \mathrm{Me}) ; 1.55\left(s, \mathrm{Me}^{*}\right) ; 2.19-$ $2.31\left(m, \mathrm{H}_{\mathrm{a}}-\mathrm{C}(2), \mathrm{H}-\mathrm{C}\left(3^{\prime}\right), \mathrm{H}-\mathrm{C}\left(3^{\prime *}\right)\right) ; 2.38\left(d d,{ }^{2} J\left(2 \mathrm{a}^{*}, 2 \mathrm{~b}^{*}\right)=16.8,{ }^{3} \mathrm{~J}\left(2 \mathrm{a}^{*}, 3^{\prime *}\right)=4.6, \mathrm{H}_{\mathrm{a}}-\mathrm{C}\left(2^{*}\right)\right) ; 2.54$ $\left(d d,{ }^{2} J(2 \mathrm{~b}, 2 \mathrm{a})=16.1,{ }^{3} J\left(2 \mathrm{~b}, 3^{\prime}\right)=9.5, \mathrm{H}_{\mathrm{b}}-\mathrm{C}(2)\right) ; 2.71\left(d d,{ }^{2} J\left(2 \mathrm{~b}^{*}, 2 \mathrm{a}^{*}\right)=16.8,{ }^{3} J\left(2 \mathrm{~b}^{*}, 3^{\prime *}\right)=9.9, \mathrm{H}_{\mathrm{b}}-\mathrm{C}\left(2^{*}\right)\right)$; $3.64(s, \mathrm{MeO} *) ; 3.68(s, \mathrm{MeO}) ; 4.34\left(d d,{ }^{3} J\left(2^{\prime *}, 3^{\prime *}\right)=10.2,{ }^{3} J\left(2^{\prime *}, 6^{*} *\right)=8.5, \mathrm{H}-\mathrm{C}\left(2^{\prime *}\right)\right) ; 4.53\left(d d,{ }^{3} J\left(2^{\prime}, 3^{\prime}\right)=\right.$ 9.7, $\left.{ }^{3} J\left(2^{\prime}, 6^{\prime}\right)=9.7, \mathrm{H}-\mathrm{C}\left(2^{\prime}\right)\right) ; 4.79\left(d d,{ }^{3} J\left(4^{\prime}, 5^{\prime}\right)=3.9,{ }^{3} J\left(4^{\prime}, 3^{\prime}\right)=3.9, \mathrm{H}-\mathrm{C}\left(4^{\prime}\right)\right) ; 4.84\left(d d,{ }^{3} J\left(4^{\prime} * 5^{\prime *}\right)=4.1\right.$, $\left.{ }^{3} J\left(4^{\prime *}, 3^{\prime *}\right)=4.1, \mathrm{H}-\mathrm{C}\left(4^{\prime *}\right)\right) ; 5.69\left(d d,{ }^{3} J\left(6^{\prime}, 7^{\prime}\right)=11.4,{ }^{3} J\left(6^{\prime}, 2^{\prime}\right)=9.7, \mathrm{H}-\mathrm{C}\left(6^{\prime}\right)\right) ; 5.90\left(d,{ }^{3} J\left(5^{\prime}, 4^{\prime}\right)=3.9\right.$, $\left.\mathrm{H}-\mathrm{C}\left(5^{\prime}\right)\right)$; 5.90-5.92 $\left(m, \mathrm{H}-\mathrm{C}\left(5^{\prime *}\right)\right) ; 6.18\left(d d,{ }^{3} J\left(6^{\prime *}, 7^{\prime *}\right)=15.9,{ }^{3} J\left(6^{\prime *}, 2^{\prime *}\right)=7.8, \mathrm{H}-\mathrm{C}\left(6^{\prime *}\right)\right) ; 6.69(d$, $\left.{ }^{3} J\left(7^{\prime *}, 6^{\prime *}\right)=15.9, \mathrm{H}-\mathrm{C}\left(7^{\prime *}\right)\right) ; 6.82\left(d,{ }^{3} J\left(7^{\prime}, 6^{\prime}\right)=11.4, \mathrm{H}-\mathrm{C}\left(7^{\prime}\right)\right) ; 7.46-7.50\left(m, \mathrm{H}-\mathrm{C}\left(3^{\prime \prime}\right), \mathrm{H}-\mathrm{C}\left(5^{\prime \prime}\right)\right.$, $\left.\mathrm{H}-\mathrm{C}\left(3^{\prime \prime *}\right), \mathrm{H}-\mathrm{C}\left(5^{\prime \prime *}\right)\right) ; 7.56-7.61\left(m, \mathrm{H}-\mathrm{C}\left(2^{\prime \prime}\right), \mathrm{H}-\mathrm{C}\left(6^{\prime \prime}\right), \mathrm{H}-\mathrm{C}\left(2^{\prime \prime *}\right), \mathrm{H}-\mathrm{C}\left(6^{\prime \prime *}\right)\right) \cdot{ }^{13} \mathrm{C}-\mathrm{NMR}\left(\mathrm{CDCl}_{3}\right.$, $\left.100 \mathrm{MHz})^{1}\right): 26.3\left(\mathrm{Me}^{*}\right) ; 26.5(\mathrm{Me}) ; 26.6\left(\mathrm{Me}, \mathrm{Me}^{*}\right) ; 29.1(\mathrm{C}(2)) ; 29.2\left(\mathrm{C}\left(2^{*}\right)\right) ; 45.9\left(\mathrm{C}\left(3^{\prime *}\right)\right) ; 46.6$ $\left(\mathrm{C}\left(3^{\prime}\right)\right) ; 51.8(\mathrm{MeO}, \mathrm{MeO} *) ; 75.7\left(\mathrm{C}\left(2^{\prime}\right)\right) ; 80.8\left(\mathrm{C}\left(4^{\prime}\right), \mathrm{C}\left(4^{\prime} *\right)\right) ; 81.5\left(\mathrm{C}\left(2^{\prime *}\right)\right) ; 105.0\left(\mathrm{C}\left(5^{\prime}\right), \mathrm{C}\left(5^{\prime} *\right)\right) ; 111.8$ $\left(\mathrm{Me}_{2} \mathrm{C}, \mathrm{Me}_{2} \mathrm{C}^{*}\right) ; 125.3\left(q,{ }^{3} \mathrm{~J}(\mathrm{C}, \mathrm{F})=3.8, \mathrm{C}\left(3^{\prime \prime}\right), \mathrm{C}\left(5^{\prime \prime}\right)\right) ; 125.6\left(q,{ }^{3} \mathrm{~J}(\mathrm{C}, \mathrm{F})=3.9, \mathrm{C}\left(3^{\prime \prime *}\right), \mathrm{C}\left(5^{\prime \prime *}\right)\right) ; 126.8$ $\left(\mathrm{C}\left(6^{\prime}\right)\right) ; 128.9\left(\mathrm{C}\left(6^{\prime *}\right)\right) ; 129.1\left(\mathrm{C}\left(2^{\prime \prime}\right), \mathrm{C}\left(6^{\prime \prime}\right)\right) ; 129.8\left(\mathrm{C}\left(2^{\prime \prime *}\right), \mathrm{C}\left(6^{\prime \prime *}\right)\right) ; 129.8\left[q,{ }^{2} J_{\mathrm{C} F}=32.3, \mathrm{C}\left(4^{\prime \prime}\right)\right) ; 132.7$ $\left(\mathrm{C}\left(7^{\prime *}\right)\right) ; 135.4\left(\mathrm{C}\left(7^{\prime}\right)\right) ; 139.5\left(\mathrm{C}\left(1^{\prime \prime}\right)\right) ; 172.3(\mathrm{C}(1))$. Anal. calc. for $\mathrm{C}_{19} \mathrm{H}_{21} \mathrm{~F}_{3} \mathrm{O}_{5}(386.37)$ : C 59.07, H 5.48; found: C 58.88, H 5.31.

Preparation of $\mathbf{4 a}-\mathbf{4 c}$. To a soln. of $\mathbf{3 a}(0.87 \mathrm{~g}, 2.7 \mathrm{mmol}), \mathbf{3 b}(0.87 \mathrm{~g} ; 2.6 \mathrm{mmol})$, or $\mathbf{3 c}(1.10 \mathrm{~g}$; $2.8 \mathrm{mmol}$ ) in AcOEt $(120 \mathrm{ml}), 60 \mathrm{mg}$ of $\mathrm{Pd} / \mathrm{C} 10 \%$ were added. The suspension was shaken under $\mathrm{H}_{2}$ for 
$20 \mathrm{~h}$ at r.t. The mixture was filtered, and the solvent was removed i.v. to yield the esters $\mathbf{4 a}(0.81 \mathrm{~g}, 92 \%)$, 4b $(0.82 \mathrm{~g}, 94 \%)$, and $4 \mathbf{c}(1.04 \mathrm{~g}, 94 \%)$, resp.

Methyl 2-[(2R,3R,4R,5R)-2,3,4,5-Tetrahydro-4,5-(isopropylidenedioxy)-2-(2-(phenylethyl)furan-3$y$ l)acetate (4a). White crystals. $R_{\mathrm{f}}$ (hexane/AcOEt $3: 1$ ) 0.55. M.p. 77.8-79.5 (hexane/AcOEt $3: 1$ ). $[\alpha]_{\mathrm{D}}^{24}=+173.1\left(c=1.04, \mathrm{CH}_{2} \mathrm{Cl}_{2}\right)$. IR $(\mathrm{KBr}): 3028,2985,2943,2867,1734,1602,1461,1440,1387,1375$, 1212, 1136, 1091, 1011, 871, 703. ${ }^{1} \mathrm{H}-\mathrm{NMR}\left(\mathrm{CDCl}_{3}, 400 \mathrm{MHz}\right): 1.32(s, \mathrm{Me}) ; 1.47(\mathrm{~s}, \mathrm{Me}) ; 1.67-1.77(\mathrm{~m}$, $\left.\mathrm{H}_{\mathrm{a}}-\mathrm{C}\left(6^{\prime}\right)\right) ; 1.82-1.90\left(m, \mathrm{H}_{\mathrm{b}}-\mathrm{C}\left(6^{\prime}\right)\right) ; 2.04-2.13\left(m, \mathrm{H}-\mathrm{C}\left(3^{\prime}\right)\right) ; 2.28\left(d d,{ }^{2} J(2 \mathrm{a}, 2 \mathrm{~b})=16.9,{ }^{3} J\left(2 \mathrm{a}, 3^{\prime}\right)=4.3\right.$, $\left.\mathrm{H}_{\mathrm{a}}-\mathrm{C}(2)\right) ; 2.58-2.72\left(m, \mathrm{H}_{\mathrm{a}}-\mathrm{C}\left(7^{\prime}\right)\right) ; 2.61\left(d d,{ }^{2} J(2 \mathrm{~b}, 2 \mathrm{a})=16.9,{ }^{3} J\left(2 \mathrm{~b}, 3^{\prime}\right)=10.2, \mathrm{H}_{\mathrm{b}}-\mathrm{C}(2)\right) ; 2.84-2.91$ $\left(m, \mathrm{H}_{\mathrm{b}}-\mathrm{C}\left(7^{\prime}\right)\right) ; 3.69(\mathrm{~s}, \mathrm{MeO}) ; 3.76-3.81\left(m, \mathrm{H}-\mathrm{C}\left(2^{\prime}\right)\right) ; 4.76\left(t,{ }^{3} J\left(4^{\prime}, 5^{\prime}\right)=4.0,{ }^{3} J\left(4^{\prime}, 3^{\prime}\right)=4.0, \mathrm{H}-\mathrm{C}\left(4^{\prime}\right)\right)$; $5.85\left(d,{ }^{3} J\left(5^{\prime}, 4^{\prime}\right)=4.0, \mathrm{H}-\mathrm{C}\left(5^{\prime}\right)\right) ; 7.15-7.20\left(m, \mathrm{H}-\mathrm{C}\left(3^{\prime \prime}\right), \mathrm{H}-\mathrm{C}\left(4^{\prime \prime}\right), \mathrm{H}-\mathrm{C}\left(5^{\prime \prime}\right)\right) ; 7.25-7.29(m$, $\left.\mathrm{H}-\mathrm{C}\left(2^{\prime \prime}\right), \mathrm{H}-\mathrm{C}\left(6^{\prime \prime}\right)\right) .{ }^{13} \mathrm{C}-\mathrm{NMR}\left(\mathrm{CDCl}_{3}, 100 \mathrm{MHz}\right): 26.4(\mathrm{Me}) ; 26.5(\mathrm{Me}) ; 29.5(\mathrm{C}(2)) ; 32.3\left(\mathrm{C}\left(7^{\prime}\right)\right)$; $34.5\left(\mathrm{C}\left(6^{\prime}\right)\right) ; 44.8\left(\mathrm{C}\left(3^{\prime}\right)\right) ; 51.8(\mathrm{MeO}) ; 79.5\left(\mathrm{C}\left(2^{\prime}\right)\right) ; 81.2\left(\mathrm{C}\left(4^{\prime}\right)\right) ; 104.7\left(\mathrm{C}\left(5^{\prime}\right)\right) ; 111.4\left(\mathrm{CMe}_{2}\right) ; 125.9(\mathrm{C}(4)$ of $\mathrm{Ph}) ; 128.4(\mathrm{C}(2), \mathrm{C}(3), \mathrm{C}(5), \mathrm{C}(6)$ of $\mathrm{Ph}) ; 141.8(\mathrm{C}(1)$ of $\mathrm{Ph}) ; 172.6(\mathrm{C}(1))$. Anal. calc. for $\mathrm{C}_{18} \mathrm{H}_{24} \mathrm{O}_{5}$ (320.39): C 67.48, H 7.55; found: C 67.84, H 7.73.

Methyl 2-\{(2R,3R,4R,5R)-2,3,4,5-Tetrahydro-4,5-(isopropylidenedioxy)-2-[2-(4-methylphenyl)ethyl]furan-3-yllacetate (4b). White crystals. $R_{\mathrm{f}}$ (hexane/AcOEt $3: 1$ ) 0.60. M.p. 56.7-58.0 $0^{\circ}$ (hexane/ AcOEt $3: 1) .[\alpha]_{\mathrm{D}}^{21}=+225.5\left(c=1.02, \mathrm{CH}_{2} \mathrm{Cl}_{2}\right)$. IR $(\mathrm{KBr}): 2985,2943,2859,1737,1514,1434,1373,1335$, 1255, 1211, 1140, 1017, 978, 879, 807, 532. ${ }^{1} \mathrm{H}-\mathrm{NMR}\left(\mathrm{CDCl}_{3}, 200 \mathrm{MHz}\right): 1.32(\mathrm{~s}, \mathrm{Me}) ; 1.47(\mathrm{~s}, \mathrm{Me}) ; 1.60-$ $1.92\left(m, \mathrm{CH}_{2}\left(6^{\prime}\right)\right) ; 2.01-2.16\left(m, \mathrm{H}-\mathrm{C}\left(3^{\prime}\right)\right) ; 2.28\left(d d,{ }^{2} J(2 \mathrm{a}, 2 \mathrm{~b})=16.9,{ }^{3} J\left(2 \mathrm{a}, 3^{\prime}\right)=4.0, \mathrm{H}_{\mathrm{a}}-\mathrm{C}(2)\right) ; 2.31(s$, $\mathrm{Me}-\mathrm{C}(4)$ of $\left.\mathrm{C}_{6} \mathrm{H}_{4}\right) ; 2.60-2.71\left(m, \mathrm{H}_{\mathrm{a}}-\mathrm{C}\left(7^{\prime}\right)\right) ; 2.63\left(d d,{ }^{2} J(2 \mathrm{~b}, 2 \mathrm{a})=16.9,{ }^{3} J\left(2 \mathrm{~b}, 3^{\prime}\right)=10.3, \mathrm{H}_{\mathrm{b}}-\mathrm{C}(2)\right)$; $2.76-2.91\left(m, \mathrm{H}_{\mathrm{b}}-\mathrm{C}\left(7^{\prime}\right)\right) ; 3.69(\mathrm{~s}, \mathrm{MeO}) ; 3.73-3.84\left(m, \mathrm{H}-\mathrm{C}\left(2^{\prime}\right)\right) ; 4.76\left(t,{ }^{3} J\left(4^{\prime}, 5^{\prime}\right)=4.0,{ }^{3} J\left(4^{\prime}, 3^{\prime}\right)=4.0\right.$, $\left.\mathrm{H}-\mathrm{C}\left(4^{\prime}\right)\right) ; 5.84\left(d,{ }^{3} J\left(5^{\prime}, 4^{\prime}\right)=4.0, \mathrm{H}-\mathrm{C}\left(5^{\prime}\right)\right) ; 7.08$ (br. $\left.s, \mathrm{H}-\mathrm{C}\left(2^{\prime \prime}\right), \mathrm{H}-\mathrm{C}\left(3^{\prime \prime}\right), \mathrm{H}-\mathrm{C}\left(5^{\prime \prime}\right), \mathrm{H}-\mathrm{C}\left(6^{\prime \prime}\right)\right)$. ${ }^{13} \mathrm{C}-\mathrm{NMR}\left(\mathrm{CDCl}_{3}, 50 \mathrm{MHz}\right): 21.0\left(\mathrm{Me}-\mathrm{C}(4)\right.$ of $\left.\mathrm{C}_{6} \mathrm{H}_{4}\right) ; 26.4(\mathrm{Me}) ; 26.6(\mathrm{Me}) ; 29.5(\mathrm{C}(2)) ; 31.8\left(\mathrm{C}\left(7^{\prime}\right)\right)$; $34.6\left(\mathrm{C}\left(6^{\prime}\right)\right) ; 44.8\left(\mathrm{C}\left(3^{\prime}\right)\right) ; 51.8(\mathrm{MeO}) ; 79.5\left(\mathrm{C}\left(2^{\prime}\right)\right) ; 81.1\left(\mathrm{C}\left(4^{\prime}\right)\right) ; 104.7\left(\mathrm{C}\left(5^{\prime}\right)\right) ; 111.4\left(\mathrm{Me}_{2} \mathrm{C}\right) ; 128.8$ $\left(\mathrm{C}(2), \mathrm{C}(6)\right.$ of $\left.\mathrm{C}_{6} \mathrm{H}_{4}\right) ; 129.1\left(\mathrm{C}(3), \mathrm{C}(5)\right.$ of $\left.\mathrm{C}_{6} \mathrm{H}_{4}\right) ; 135.3\left(\mathrm{C}(4)\right.$ of $\left.\mathrm{C}_{6} \mathrm{H}_{4}\right) ; 138.8\left(\mathrm{C}(1)\right.$ of $\left.\mathrm{C}_{6} \mathrm{H}_{4}\right) ; 172.6$ $(\mathrm{C}(1))$. Anal. calc. for $\mathrm{C}_{19} \mathrm{H}_{26} \mathrm{O}_{5}$ (334.41): C 68.24, $\mathrm{H} 7.84$; found: $\mathrm{C} 68.64, \mathrm{H} 7.74$.

Methyl 2-\{(2R,3R,4R,5R)-2,3,4,5-Tetrahydro-4,5-(isopropylidenedioxy)-2-\{2-[4-(trifluoromethyl)phenyl]ethylffuran-3-yl\}acetate (4c). White crystals. $R_{\mathrm{f}}$ (hexane/AcOEt $3: 1$ ) 0.49. M.p. 71.7-74.2 (hexane/AcOEt $3: 1)$. $[\alpha]_{\mathrm{D}}^{26}=+203.6\left(c=1.67, \mathrm{CH}_{2} \mathrm{Cl}_{2}\right)$. IR (KBr): 2992, 2961, 2864, 1735, 1617, 1431 , 1325, 1209, 1164, 1118, 1017, 895. ${ }^{1} \mathrm{H}-\mathrm{NMR}\left(\mathrm{CDCl}_{3}, 400 \mathrm{MHz}\right): 1.32(\mathrm{~s}, \mathrm{Me}) ; 1.46(\mathrm{~s}, \mathrm{Me}) ; 1.71-1.77(m$, $\left.\mathrm{H}_{\mathrm{a}}-\mathrm{C}\left(6^{\prime}\right)\right) ; 1.83-1.88\left(m, \mathrm{H}_{\mathrm{b}}-\mathrm{C}\left(6^{\prime}\right)\right) ; 2.06-2.14\left(m, \mathrm{H}-\mathrm{C}\left(3^{\prime}\right)\right) ; 2.27\left(d d,{ }^{2} J(2 \mathrm{a}, 2 \mathrm{~b})=16.9,{ }^{3} J\left(2 \mathrm{a}, 3^{\prime}\right)=4.5\right.$, $\left.\mathrm{H}_{\mathrm{a}}-\mathrm{C}(2)\right) ; 2.64\left(d d,{ }^{2} J(2 \mathrm{~b}, 2 \mathrm{a})=16.9,{ }^{3} J\left(2 \mathrm{~b}, 3^{\prime}\right)=9.8, \mathrm{H}_{\mathrm{b}}-\mathrm{C}(2)\right) ; 2.72-2.79\left(m, \mathrm{H}_{\mathrm{a}}-\mathrm{C}\left(7^{\prime}\right)\right) ; 2.89-2.96(m$, $\left.\mathrm{H}_{\mathrm{b}}-\mathrm{C}\left(7^{\prime}\right)\right)$; $3.69(\mathrm{~s}, \mathrm{MeO}) ; 3.74-3.79\left(m, \mathrm{H}-\mathrm{C}\left(2^{\prime}\right)\right) ; 4.76\left(d d,{ }^{3} J\left(4^{\prime}, 5^{\prime}\right)=4.0,{ }^{3} J\left(4^{\prime}, 3^{\prime}\right)=4.0, \mathrm{H}-\mathrm{C}\left(4^{\prime}\right)\right)$; $5.84\left(d,{ }^{3} J\left(5^{\prime}, 4^{\prime}\right)=4.0, \mathrm{H}-\mathrm{C}\left(5^{\prime}\right)\right) ; 7.30\left(d,{ }^{3} J\left(2^{\prime \prime}, 3^{\prime \prime}\right)=8.0,{ }^{3} J\left(6^{\prime \prime}, 5^{\prime \prime}\right)=8.0, \mathrm{H}-\mathrm{C}\left(2^{\prime \prime}\right), \mathrm{H}-\mathrm{C}\left(6^{\prime \prime}\right)\right) ; 7.53(d$, $\left.{ }^{3} J\left(3^{\prime \prime}, 2^{\prime \prime}\right)=8.0,{ }^{3} J\left(5^{\prime \prime}, 6^{\prime \prime}\right)=8.0, \mathrm{H}-\mathrm{C}\left(3^{\prime \prime}\right), \mathrm{H}-\mathrm{C}\left(5^{\prime \prime}\right)\right) \cdot{ }^{13} \mathrm{C}-\mathrm{NMR}\left(\mathrm{CDCl}_{3}, 100 \mathrm{MHz}\right): 26.3(\mathrm{Me}) ; 26.5$ $(\mathrm{Me}) ; 29.5(\mathrm{C}(2)) ; 32.0\left(\mathrm{C}\left(7^{\prime}\right)\right) ; 34.0\left(\mathrm{C}\left(6^{\prime}\right)\right) ; 44.7\left(\mathrm{C}\left(3^{\prime}\right)\right) ; 51.7(\mathrm{MeO}) ; 79.2\left(\mathrm{C}\left(2^{\prime}\right)\right) ; 81.2\left(\mathrm{C}\left(4^{\prime}\right)\right) ; 104.6$ $\left(\mathrm{C}\left(5^{\prime}\right)\right) ; 111.5\left(\mathrm{Me}_{2} \mathrm{C}\right), 125.3\left(q,{ }^{3} J(\mathrm{C}, \mathrm{F})=3.7, \mathrm{C}(3), \mathrm{C}(5)\right.$ of $\left.\mathrm{C}_{6} \mathrm{H}_{4}\right) ; 128.4\left(q,{ }^{2} J(\mathrm{C}, \mathrm{F})=32.1, \mathrm{C}(4)\right.$ of $\left.\mathrm{C}_{6} \mathrm{H}_{4}\right) ; 128.7\left(\mathrm{C}(2), \mathrm{C}(6)\right.$ of $\left.\mathrm{C}_{6} \mathrm{H}_{4}\right) ; 145.9\left(\mathrm{C}(1)\right.$ of $\left.\mathrm{C}_{6} \mathrm{H}_{4}\right) ; 172.5(\mathrm{C}(1))$. Anal. calc. for $\mathrm{C}_{19} \mathrm{H}_{23} \mathrm{~F}_{3} \mathrm{O}_{5}$ (388.38): C 58.76, H 5.97; found: C 58.87, H 6.13.

Preparation of $\mathbf{5 a}-\mathbf{5 c}$. To a stirring soln. of the esters $4 \mathbf{a}(0.74 \mathrm{~g}, 2.3 \mathrm{mmol}), \mathbf{4 b}(0.75 \mathrm{~g}, 2.2 \mathrm{mmol})$, or $4 \mathbf{c}(0.94 \mathrm{~g}, 2.4 \mathrm{mmol})$ in 1,4-dioxane $(35 \mathrm{ml})$, an aq. soln. of $2 \% \mathrm{H}_{2} \mathrm{SO}_{4}(v / v ; 16 \mathrm{ml})$ was added. The mixture was stirred under reflux for $3 \mathrm{~h}$. The product was extracted with $\mathrm{Et}_{2} \mathrm{O}(200 \mathrm{ml})$. The org. phase was washed with dist. $\mathrm{H}_{2} \mathrm{O}(35 \mathrm{ml})$ and with sat. aq. $\mathrm{NaHCO}_{3}$ soln. $(35 \mathrm{ml})$, dried $\left(\mathrm{Na}_{2} \mathrm{SO}_{4}\right)$, and concentrated i.v. The crude material was purified by $\mathrm{CC}\left(\mathrm{SiO}_{2}\right.$; hexane/AcOEt $\left.1: 1\right)$ to yield the mixture of epimers $5 \mathbf{a}(0.54 \mathrm{~g}, 94 \%), \mathbf{5 b}(0.57 \mathrm{~g}, 97 \%)$, and $\mathbf{5 c}(0.69 \mathrm{~g}, 90 \%)$, resp.

(1R,5R,6R,8R)- and (1R,5R,6R,8S)-8-Hydroxy-6-(2-phenylethyl)-2,7-dioxabicyclo[3.3.0]octan-3one (5a). Colorless oil. $R_{\mathrm{f}}$ (hexane/AcOEt 1:1) 0.43. IR (film): 3430, 3032, 2936, 2864, 1781, 1636, 1496, 1455, 1362, 1167, 1047, 702. $\left.{ }^{1} \mathrm{H}-\mathrm{NMR}\left(\mathrm{CDCl}_{3}, 400 \mathrm{MHz}\right)^{2}\right): 1.88-2.00\left(m, \mathrm{H}_{\mathrm{a}}-\mathrm{C}(9), \mathrm{CH}_{2}\left(9^{*}\right)\right)$; 2.07-2.17 (m, $\left.\mathrm{H}_{\mathrm{b}}-\mathrm{C}(9)\right) ; 2.40\left(d d,{ }^{2} J(4 \mathrm{a}, 4 \mathrm{~b})=18.0,{ }^{3} J(4 \mathrm{a}, 5)=1.6, \mathrm{H}_{\mathrm{a}}-\mathrm{C}(4)\right) ; 2.42-2.49\left(m, \mathrm{H}_{\mathrm{a}}-\mathrm{C}\left(4^{*}\right)\right)$; 2.64-2.83 (m, $\left.\mathrm{CH}_{2}(10), \mathrm{H}_{\mathrm{b}}-\mathrm{C}\left(4^{*}\right), \mathrm{H}-\mathrm{C}\left(5^{*}\right), \mathrm{CH}_{2}\left(10^{*}\right)\right) ; 2.77\left(d d,{ }^{2} J(4 \mathrm{~b}, 4 \mathrm{a})=18.0,{ }^{3} J(4 \mathrm{~b}, 5)=9.2\right.$,

2) The signals marked '*' are due to the $\alpha$-epimer. All the others refer to the major $\beta$-epimer $(\alpha / \beta$ 1:2). 
$\left.\mathrm{H}_{\mathrm{b}}-\mathrm{C}(4)\right) ; 2.88-2.94(m, \mathrm{H}-\mathrm{C}(5)) ; 3.40$ (br. $\left.s, \mathrm{OH}\right) ; 3.70$ (br. $\left.s, \mathrm{OH}^{*}\right) ; 3.94\left(d d d,{ }^{3} J(6,5)=8.2,{ }^{3} J(6,9 \mathrm{a})=\right.$ $\left.5.2,{ }^{3} J(6,9 \mathrm{~b})=5.2, \mathrm{H}-\mathrm{C}(6)\right) ; 3.98-4.03\left(m, \mathrm{H}-\mathrm{C}\left(6^{*}\right)\right) ; 4.87\left(d,{ }^{3} J(1,5)=6.3, \mathrm{H}-\mathrm{C}(1)\right) ; 4.87-4.90(m$, $\left.\mathrm{H}-\mathrm{C}\left(1^{*}\right)\right) ; 5.53\left(d,{ }^{3} J\left(8^{*}, 1^{*}\right)=4.0, \mathrm{H}-\mathrm{C}\left(8^{*}\right)\right) ; 5.56(s, \mathrm{H}-\mathrm{C}(8)) ; 7.17-7.31\left(m, \mathrm{H}-\mathrm{C}\left(2^{\prime}\right), \mathrm{H}-\mathrm{C}\left(3^{\prime}\right)\right.$, $\left.\mathrm{H}-\mathrm{C}\left(4^{\prime}\right), \mathrm{H}-\mathrm{C}\left(5^{\prime}\right), \mathrm{H}-\mathrm{C}\left(6^{\prime}\right), \mathrm{H}-\mathrm{C}\left(2^{\prime *}\right), \mathrm{H}-\mathrm{C}\left(3^{\prime *}\right), \mathrm{H}-\mathrm{C}\left(4^{\prime} *\right), \mathrm{H}-\mathrm{C}\left(5^{\prime *}\right), \mathrm{H}-\mathrm{C}\left(6^{\prime *}\right)\right) .{ }^{13} \mathrm{C}-\mathrm{NMR}$ $\left.\left(\mathrm{CDCl}_{3}, 100 \mathrm{MHz}\right)^{2}\right): 32.0\left(\mathrm{C}\left(10^{*}\right)\right) ; 32.3(\mathrm{C}(10)) ; 33.3\left(\mathrm{C}\left(4^{*}\right)\right) ; 33.9(\mathrm{C}(4)) ; 36.4\left(\mathrm{C}\left(9^{*}\right)\right) ; 39.2(\mathrm{C}(9))$; $42.1\left(\mathrm{C}\left(5^{*}\right)\right) ; 42.6(\mathrm{C}(5)) ; 82.2\left(\mathrm{C}\left(1^{*}\right)\right) ; 82.7\left(\mathrm{C}\left(6^{*}\right)\right) ; 87.3(\mathrm{C}(6)) ; 88.3(\mathrm{C}(1)) ; 95.6\left(\mathrm{C}\left(8^{*}\right)\right) ; 101.1(\mathrm{C}(8))$; $126.2\left(\mathrm{C}\left(4^{\prime}\right), \mathrm{C}\left(4^{\prime *}\right)\right) ; 128.3\left(\mathrm{C}\left(2^{\prime *}\right), \mathrm{C}\left(6^{\prime *}\right)\right) ; 128.4\left(\mathrm{C}\left(2^{\prime}\right), \mathrm{C}\left(6^{\prime}\right)\right) ; 128.6\left(\mathrm{C}\left(3^{\prime}\right), \mathrm{C}\left(5^{\prime}\right), \mathrm{C}\left(3^{\prime *}\right), \mathrm{C}\left(5^{\prime *}\right)\right)$; $140.9\left(\mathrm{C}\left(1^{\prime *}\right)\right) ; 141.0\left(\mathrm{C}\left(1^{\prime}\right)\right) ; 175.7(\mathrm{C}(3))$; $176.6\left(\mathrm{C}\left(3^{*}\right)\right)$. Anal. calc. for $\mathrm{C}_{14} \mathrm{H}_{16} \mathrm{O}_{4}(248.28): \mathrm{C} 67.73, \mathrm{H}$ 6.50; found: C $67.83, \mathrm{H} 6.56$.

$(1 \mathrm{R}, 5 \mathrm{R}, 6 \mathrm{R}, 8 \mathrm{R})-\quad$ and $\quad(1 \mathrm{R}, 5 \mathrm{R}, 6 \mathrm{R}, 8 \mathrm{~S})-8-$ Hydroxy-2,7-6-[2-(4-methylphenyl)ethyl)-dioxabicyclo[3.3.0]octan-3-one (5b). White crystals. $R_{\mathrm{f}}$ (hexane/AcOEt 1:1) 0.47. M.p. 77.0-78.5 (hexane/ AcOEt 1:1). IR (KBr): 3419, 3042, 2950, 2910, 2860, 1755, 1515, 1451, 1414, 1198, 1179, 1063, 1048, 973, 907, 802, 778. $\left.{ }^{1} \mathrm{H}-\mathrm{NMR}\left(\mathrm{CDCl}_{3}, 200 \mathrm{MHz}\right)^{2}\right): 1.84-1.97\left(m, \mathrm{H}_{\mathrm{a}}-\mathrm{C}(9), \mathrm{CH}_{2}\left(9^{*}\right)\right) ; 2.00-2.19(m$, $\left.\mathrm{H}_{\mathrm{b}}-\mathrm{C}(9)\right) ; 2.32\left(s, \mathrm{Me}, \mathrm{Me}^{*}\right) ; 2.35-2.48\left(m, \mathrm{H}_{\mathrm{a}}-\mathrm{C}(4), \mathrm{H}_{\mathrm{a}}-\mathrm{C}(4 *)\right) ; 2.69-2.89\left(m, \mathrm{H}_{\mathrm{b}}-\mathrm{C}(4), \mathrm{CH}_{2}(10)\right.$, $\left.\mathrm{H}_{\mathrm{b}}-\mathrm{C}(4 *), \mathrm{H}-\mathrm{C}\left(5^{*}\right), \mathrm{CH}_{2}\left(10^{*}\right), \mathrm{OH}\right) ; 2.84-2.95\left(m, \mathrm{H}-\mathrm{C}(5), \mathrm{OH}^{*}\right) ; 3.93\left(d d d,{ }^{3} J(6,5)=8.1,{ }^{3} \mathrm{~J}(6,9 \mathrm{a})=\right.$ $\left.5.2,{ }^{3} \mathrm{~J}(6,9 \mathrm{~b})=5.2, \mathrm{H}-\mathrm{C}(6)\right) ; 3.96-4.04\left(m, \mathrm{H}-\mathrm{C}\left(6^{*}\right)\right) ; 4.87\left(d,{ }^{3} J(1,5)=6.2, \mathrm{H}-\mathrm{C}(1)\right) ; 4.86-4.91(m$, $\left.\mathrm{H}-\mathrm{C}\left(1^{*}\right)\right) ; 5.53\left(d,{ }^{3} \mathrm{~J}\left(8^{*}, 1^{*}\right)=4.1, \mathrm{H}-\mathrm{C}\left(8^{*}\right)\right) ; 5.55(s, \mathrm{H}-\mathrm{C}(8)) ; 7.04-7.13\left(m, \mathrm{H}-\mathrm{C}\left(2^{\prime}\right), \mathrm{H}-\mathrm{C}\left(3^{\prime}\right)\right.$, $\left.\left.\mathrm{H}-\mathrm{C}\left(5^{\prime}\right), \mathrm{H}-\mathrm{C}\left(6^{\prime}\right), \mathrm{H}-\mathrm{C}\left(2^{\prime *}\right), \mathrm{H}-\mathrm{C}\left(3^{\prime *}\right), \mathrm{H}-\mathrm{C}\left(5^{\prime *}\right), \mathrm{H}-\mathrm{C}\left(6^{\prime *}\right)\right) .{ }^{13} \mathrm{C}-\mathrm{NMR}\left(\mathrm{CDCl}_{3}, 50 \mathrm{MHz}\right)^{2}\right): 20.9$ $\left(\mathrm{Me}, \mathrm{Me}^{*}\right) ; 31.4\left(\mathrm{C}\left(10^{*}\right)\right) ; 31.7(\mathrm{C}(10)) ; 33.3\left(\mathrm{C}\left(4^{*}\right)\right) ; 33.9(\mathrm{C}(4)) ; 36.5\left(\mathrm{C}\left(9^{*}\right)\right) ; 39.2(\mathrm{C}(9)) ; 42.0\left(\mathrm{C}\left(5^{*}\right)\right)$; $42.5(\mathrm{C}(5)) ; 82.2\left(\mathrm{C}\left(1^{*}\right)\right) ; 82.6\left(\mathrm{C}\left(6^{*}\right)\right) ; 87.2(\mathrm{C}(6)) ; 88.3(\mathrm{C}(1)) ; 95.5\left(\mathrm{C}\left(8^{*}\right)\right) ; 101.0(\mathrm{C}(8))$; $128.2\left(\mathrm{C}\left(2^{\prime}\right)\right.$, $\left.\mathrm{C}\left(6^{\prime}\right), \mathrm{C}\left(2^{\prime *}\right), \mathrm{C}\left(6^{\prime *}\right)\right) ; 129.2\left(\mathrm{C}\left(3^{\prime}\right), \mathrm{C}\left(5^{\prime}\right), \mathrm{C}\left(3^{\prime *}\right), \mathrm{C}\left(5^{\prime *}\right)\right) ; 135.6\left(\mathrm{C}\left(4^{\prime}\right), \mathrm{C}\left(4^{\prime *}\right)\right) ; 137.8\left(\mathrm{C}\left(1^{\prime}\right), \mathrm{C}\left(1^{\prime *}\right)\right)$; $175.7(\mathrm{C}(3))$; $176.6\left(\mathrm{C}\left(3^{*}\right)\right)$. Anal. calc. for $\mathrm{C}_{15} \mathrm{H}_{18} \mathrm{O}_{4}$ (262.31): C 68.69, H 6.92; found: C 68.85, H 6.94.

$(1 \mathrm{R}, 5 \mathrm{R}, 6 \mathrm{R}, 8 \mathrm{R})$ - and $(1 \mathrm{R}, 5 \mathrm{R}, 6 \mathrm{R}, 8 \mathrm{~S})-8$-Hydroxy-6-[2-(4-(trifluoromethyl)phenyl]ethyl]-2,7-dioxabicyclo[3.3.0]octan-3-one (5c). White crystals. $R_{\mathrm{f}}$ (hexane/AcOEt 1:1) 0.42. M.p. 102.5-105.2 (hexane/ AcOEt 1:1). IR (KBr): 3526, 3382, 2959, 2872, 1780, 1746, 1618, 1419, 1322, 1168, 1130, 1067, 1019, 963 , 902, 631. $\left.{ }^{1} \mathrm{H}-\mathrm{NMR}\left(\mathrm{CDCl}_{3}, 400 \mathrm{MHz}\right)^{2}\right): 1.90-1.99\left(m, \mathrm{H}_{\mathrm{a}}-\mathrm{C}(9), \mathrm{CH}_{2}\left(9^{*}\right)\right) ; 2.07-2.14\left(m, \mathrm{H}_{\mathrm{b}}-\mathrm{C}(9)\right)$; $2.42\left(d d,{ }^{2} J(4 \mathrm{a}, 4 \mathrm{~b})=18.0,{ }^{3} J(4 \mathrm{a}, 5)=1.7, \mathrm{H}_{\mathrm{a}}-\mathrm{C}(4)\right) ; 2.44-2.50\left(m, \mathrm{H}_{\mathrm{a}}-\mathrm{C}(4 *)\right) ; 2.69-2.89\left(m, \mathrm{CH}_{2}(10)\right.$, $\left.\mathrm{H}_{\mathrm{b}}-\mathrm{C}\left(4^{*}\right), \mathrm{H}-\mathrm{C}\left(5^{*}\right), \mathrm{CH}_{2}\left(10^{*}\right), \mathrm{OH}\right) ; 2.81\left(d d,{ }^{2} J(4 \mathrm{~b}, 4 \mathrm{a})=18.0,{ }^{3} J(4 \mathrm{~b}, 5)=9.1, \mathrm{H}_{\mathrm{b}}-\mathrm{C}(4)\right) ; 2.89-2.95$ $(m, \mathrm{H}-\mathrm{C}(5)) ; 3.03$ (br. $\left.s, \mathrm{OH}^{*}\right) ; 3.94\left(d d d,{ }^{3} J(6,5)=8.9,{ }^{3} J(6,9 \mathrm{a})=4.7,{ }^{3} J(6,9 \mathrm{~b})=4.7, \mathrm{H}-\mathrm{C}(6)\right) ; 3.97-4.02$ $\left(m, \mathrm{H}-\mathrm{C}\left(6^{*}\right)\right) ; 4.90\left(d,{ }^{3} J(1,5)=6.4, \mathrm{H}-\mathrm{C}(1)\right) ; 4.91\left(d d,{ }^{3} J\left(1^{*}, 5^{*}\right)=8.5,{ }^{3} J\left(1^{*}, 8^{*}\right)=4.0, \mathrm{H}-\mathrm{C}\left(1^{*}\right)\right) ; 5.55$ $\left(d,{ }^{3} J\left(8^{*}, 1^{*}\right)=4.0, \mathrm{H}-\mathrm{C}\left(8^{*}\right)\right) ; 5.59(s, \mathrm{H}-\mathrm{C}(8)) ; 7.30\left(d,{ }^{3} J\left(2^{\prime}, 3^{\prime}\right)=8.0,{ }^{3} J\left(6^{\prime}, 5^{\prime}\right)=8.0,{ }^{3} J\left(2^{\prime *}, 3^{\prime *}\right)=8.0\right.$, $\left.{ }^{3} J\left(6^{*}, 5^{\prime *}\right)=8.0, \mathrm{H}-\mathrm{C}\left(2^{\prime}\right), \mathrm{H}-\mathrm{C}\left(6^{\prime}\right), \mathrm{H}-\mathrm{C}\left(2^{\prime *}\right), \mathrm{H}-\mathrm{C}\left(6^{\prime *}\right)\right) ; 7.53\left(d,{ }^{3} J\left(3^{\prime}, 2^{\prime}\right)=8.0,{ }^{3} J\left(5^{\prime}, 6^{\prime}\right)=8.0\right.$, $\left.{ }^{3} J\left(3^{\prime *}, 2^{\prime *}\right)=8.0,{ }^{3} J\left(5^{\prime *}, 6^{\prime *}\right)=8.0, \mathrm{H}-\mathrm{C}\left(3^{\prime}\right), \mathrm{H}-\mathrm{C}\left(5^{\prime}\right), \mathrm{H}-\mathrm{C}\left(3^{\prime *}\right), \mathrm{H}-\mathrm{C}\left(5^{\prime *}\right)\right) .{ }^{13} \mathrm{C}-\mathrm{NMR}\left(\mathrm{CDCl}_{3}\right.$, $\left.100 \mathrm{MHz})^{2}\right)$ : $32.0\left(\mathrm{C}\left(10^{*}\right)\right)$; $32.4(\mathrm{C}(10)) ; 33.4\left(\mathrm{C}\left(4^{*}\right)\right) ; 34.1(\mathrm{C}(4)) ; 36.3\left(\mathrm{C}\left(9^{*}\right)\right) ; 39.1(\mathrm{C}(9))$; 42.3 $\left(\mathrm{C}\left(5^{*}\right)\right) ; 42.9(\mathrm{C}(5)) ; 82.3\left(\mathrm{C}\left(1^{*}\right)\right) ; 82.5\left(\mathrm{C}\left(6^{*}\right)\right) ; 87.3(\mathrm{C}(6)) ; 88.4(\mathrm{C}(1)) ; 95.9\left(\mathrm{C}\left(8^{*}\right)\right) ; 101.3(\mathrm{C}(8)) ; 125.6$ $\left(q,{ }^{3} J(\mathrm{C}, \mathrm{F})=3.6, \mathrm{C}\left(3^{\prime}\right), \mathrm{C}\left(5^{\prime}\right), \mathrm{C}\left(3^{\prime *}\right), \mathrm{C}\left(5^{\prime *}\right)\right) ; 128.8\left(\mathrm{C}\left(2^{\prime}\right), \mathrm{C}\left(6^{\prime}\right)\right) ; 128.8\left(q,{ }^{2} J(\mathrm{C}, \mathrm{F})=33.0, \mathrm{C}\left(4^{\prime}\right)\right.$, $\left.\mathrm{C}\left(4^{\prime *}\right)\right) ; 128.9\left(\mathrm{C}\left(2^{\prime *}\right), \mathrm{C}\left(6^{\prime *}\right)\right) ; 145.2\left(\mathrm{C}\left(1^{\prime *}\right)\right) ; 145.4\left(\mathrm{C}\left(1^{\prime}\right)\right) ; 175.6(\mathrm{C}(3)) ; 176.3\left(\mathrm{C}\left(3^{*}\right)\right)$. Anal. calc. for $\mathrm{C}_{15} \mathrm{H}_{15} \mathrm{~F}_{3} \mathrm{O}_{4}$ (316.28): C 56.96, H 4.78; found: C 57.11, $\mathrm{H} 4.96$.

Preparation of $\mathbf{6 a}-6 \mathbf{c}$. The Jones reagent was prepared with $\mathrm{CrO}_{3}(2.67 \mathrm{~g})$, concentrated $\mathrm{H}_{2} \mathrm{SO}_{4}$ ( $2.3 \mathrm{ml}$ ), and distilled $\mathrm{H}_{2} \mathrm{O}$ (up to $10.0 \mathrm{ml}$ ). A portion of of this soln. (1.4 ml) was added to a stirring soln. of the compounds $\mathbf{5 a}(0.45 \mathrm{~g}, 1.8 \mathrm{mmol}), \mathbf{5 b}(0.50 \mathrm{~g}, 1.9 \mathrm{mmol})$, or $\mathbf{5 c}(0.59 \mathrm{~g}, 1.9 \mathrm{mmol})$ in acetone $(25 \mathrm{ml})$. After $5 \mathrm{~min}$ at r.t., another portion of $1.4 \mathrm{ml}$ of the Jones reagent was added, and the mixture was stirred for further $15 \mathrm{~min}$, prior to the addition of $\mathrm{MeOH}(15 \mathrm{ml})$. Distilled $\mathrm{H}_{2} \mathrm{O}(35 \mathrm{ml})$ was added, and the product was extracted with $\mathrm{Et}_{2} \mathrm{O}(4 \times 30 \mathrm{ml})$. The org. phase was washed with aq. $\mathrm{NaHCO}_{3}$ soln. $(30 \mathrm{ml})$, dried $\left(\mathrm{Na}_{2} \mathrm{SO}_{4}\right)$, and concentrated i.v. to yield the bis- $\gamma$-lactones $6 \mathbf{6}(0.36 \mathrm{~g}, 81 \%), \mathbf{6 b}(0.33 \mathrm{~g}$, $67 \%)$, and $\mathbf{6 c}(0.41 \mathrm{~g}, 70 \%)$, resp.

(1R,5R,6R)-6-(2-Phenylethyl)-2,7-dioxabicyclo[3.3.0]octan-3,8-dione (6a). White crystals. $R_{\mathrm{f}}$ (hexane/AcOEt 1:1) 0.49. M.p. 83.3-85.9 (hexane/AcOEt $1: 1)$. $[\alpha]_{\mathrm{D}}^{24}=+133.8\left(c=1.39, \mathrm{CH}_{2} \mathrm{Cl}_{2}\right)$. IR (KBr): 3080, 3026, 2932, 2866, 1794, 1774, 1602, 1455, 1423, 1311, 1249, 1221, 1169, 1132, 1022, 1000, 931, 753. ${ }^{1} \mathrm{H}-\mathrm{NMR}\left(\mathrm{CDCl}_{3}, 400 \mathrm{MHz}\right): 1.97-2.12\left(m, \mathrm{CH}_{2}(9)\right) ; 2.47\left(d d,{ }^{2} J(4 \mathrm{a}, 4 \mathrm{~b})=18.3,{ }^{3} J(4 \mathrm{a}, 5)=4.2\right.$, $\left.\mathrm{H}_{\mathrm{a}}-\mathrm{C}(4)\right) ; 2.71-2.78\left(m, \mathrm{H}_{\mathrm{a}}-\mathrm{C}(10)\right) ; 2.82-2.89\left(m, \mathrm{H}_{\mathrm{b}}-\mathrm{C}(10)\right) ; 2.88\left(d d,{ }^{2} J(4 \mathrm{~b}, 4 \mathrm{a})=18.3,{ }^{3} J(4 \mathrm{~b}, 5)=\right.$ 9.5, $\left.\mathrm{H}_{\mathrm{b}}-\mathrm{C}(4)\right) ; 3.00-3.07(m, \mathrm{H}-\mathrm{C}(5)) ; 4.31\left(d t,{ }^{3} J(6,5)=8.2,{ }^{3} J(6,9)=4.9, \mathrm{H}-\mathrm{C}(6)\right) ; 5.01\left(d,{ }^{3} J(1,5)=\right.$ 7.9, $\mathrm{H}-\mathrm{C}(1)) ; 7.18-7.33\left(m, \mathrm{H}-\mathrm{C}\left(2^{\prime}\right), \mathrm{H}-\mathrm{C}\left(3^{\prime}\right), \mathrm{H}-\mathrm{C}\left(4^{\prime}\right), \mathrm{H}-\mathrm{C}\left(5^{\prime}\right), \mathrm{H}-\mathrm{C}\left(6^{\prime}\right)\right) .{ }^{13} \mathrm{C}-\mathrm{NMR}\left(\mathrm{CDCl}_{3}\right.$, 
$100 \mathrm{MHz}): 31.3(\mathrm{C}(10)) ; 32.7(\mathrm{C}(4)) ; 37.2(\mathrm{C}(9)) ; 40.2(\mathrm{C}(5)) ; 76.9(\mathrm{C}(1)) ; 83.8(\mathrm{C}(6)) ; 126.6\left(\mathrm{C}\left(4^{\prime}\right)\right)$; $128.4\left(\mathrm{C}\left(2^{\prime}\right), \mathrm{C}\left(6^{\prime}\right)\right) ; 128.8\left(\mathrm{C}\left(3^{\prime}\right), \mathrm{C}\left(5^{\prime}\right)\right)$; $139.6\left(\mathrm{C}\left(1^{\prime}\right)\right) ; 169.8(\mathrm{C}(8))$; $173.6(\mathrm{C}(3))$. Anal. calc. for $\mathrm{C}_{14} \mathrm{H}_{14} \mathrm{O}_{4}$ (246.26): C 68.28, H 5.73; found: C 68.36, H 5.73 .

(1R,5R,6R)-6-[2-(4-Methylphenyl)ethyl]-2,7-dioxabicyclo[3.3.0]octan-3,8-dione (6b). White crystals. $R_{\mathrm{f}}$ (hexane/AcOEt 1:1) 0.51. M.p. 129.4-130.5 (hexane/AcOEt 1:1). $[\alpha]_{\mathrm{D}}^{21}=+218.5(c=1.08$, $\mathrm{CH}_{2} \mathrm{Cl}_{2}$ ). IR (KBr): 3046, 2926, 2866, 1798, 1777, 1517, 1415, 1363, 1312, 1251, 1217, 1204, 1149, 1066, 1003 , 986, 812, 722. ${ }^{1} \mathrm{H}-\mathrm{NMR}\left(\mathrm{CDCl}_{3}, 200 \mathrm{MHz}\right): 1.96-2.09\left(\mathrm{~m}, \mathrm{CH}_{2}(9)\right) ; 2.33(s, \mathrm{Me}) ; 2.47\left(d d,{ }^{2} J(4 \mathrm{a}, 4 \mathrm{~b})=\right.$ $\left.18.0,{ }^{3} J(4 \mathrm{a}, 5)=3.9, \quad \mathrm{H}_{\mathrm{a}}-\mathrm{C}(4)\right) ; 2.63-2.83\left(m, \mathrm{CH}_{2}(10)\right) ; 2.88\left(d d,{ }^{2} J(4 \mathrm{~b}, 4 \mathrm{a})=18.0,{ }^{3} J(4 \mathrm{~b}, 5)=9.4\right.$, $\left.\mathrm{H}_{\mathrm{b}}-\mathrm{C}(4)\right) ; 2.97-3.10(m, \mathrm{H}-\mathrm{C}(5)) ; 4.30\left(d t,{ }^{3} J(6,5)=7.7,{ }^{3} J(6,9 \mathrm{~b})=5.2, \mathrm{H}-\mathrm{C}(6)\right) ; 5.01\left(d,{ }^{3} J(1,5)=\right.$ 7.7, $\mathrm{H}-\mathrm{C}(1)) ; 7.05-7.15\left(m, \mathrm{H}-\mathrm{C}\left(2^{\prime}\right), \mathrm{H}-\mathrm{C}\left(3^{\prime}\right), \mathrm{H}-\mathrm{C}\left(5^{\prime}\right), \mathrm{H}-\mathrm{C}\left(6^{\prime}\right)\right) .{ }^{13} \mathrm{C}-\mathrm{NMR}\left(\mathrm{CDCl}_{3}, 50 \mathrm{MHz}\right): 21.0$ (Me); 30.8 (C(10)); $32.7(\mathrm{C}(4))$; $37.2(\mathrm{C}(9))$; 40.1 (C(5)); 76.9 (C(1)); 83.8 (C(6)); $128.3\left(\mathrm{C}\left(2^{\prime}\right), \mathrm{C}\left(6^{\prime}\right)\right)$; $129.5\left(\mathrm{C}\left(3^{\prime}\right), \mathrm{C}\left(5^{\prime}\right)\right) ; 136.2\left(\mathrm{C}\left(4^{\prime}\right)\right) ; 136.5\left(\mathrm{C}\left(1^{\prime}\right)\right) ; 169.8(\mathrm{C}(8))$; $173.6(\mathrm{C}(3))$. Anal. calc. for $\mathrm{C}_{15} \mathrm{H}_{16} \mathrm{O}_{4}$ (260.29): C 69.22, H 6.20; found: C 69.59, H 5.86.

(1R,5R,6R)-6-\{2-[4-(Trifluoromethyl)phenyl]ethyl\}-2,7-dioxabicyclo[3.3.0]octan-3,8-dione (6c). White crystals. $R_{\mathrm{f}}$ (hexane/AcOEt $1: 1$ ) 0.50. M.p. $114.5-117.1^{\circ}$ (hexane/AcOEt $1: 1$ ). $[\alpha]_{\mathrm{D}}^{26}=+146.9$ $\left(c=1.77, \mathrm{CH}_{2} \mathrm{Cl}_{2}\right)$. IR (KBr): 3048, 2964, 2922, 2872, 2844, 1779, 1617, 1420, 1324, 1213, 1119, 1065, 1020 , 935, 847, 600. ${ }^{1} \mathrm{H}-\mathrm{NMR}\left(\mathrm{CDCl}_{3}, 400 \mathrm{MHz}\right): 2.03-2.08\left(m, \mathrm{CH}_{2}(9)\right) ; 2.51\left(d d,{ }^{2} J(4 \mathrm{a}, 4 \mathrm{~b})=18.3,{ }^{3} J(4 \mathrm{a}, 5)=\right.$ 3.9, $\left.\mathrm{H}_{\mathrm{a}}-\mathrm{C}(4)\right) ; 2.78-2.86\left(m, \mathrm{H}_{\mathrm{a}}-\mathrm{C}(10)\right) ; 2.91-2.98\left(m, \mathrm{H}_{\mathrm{b}}-\mathrm{C}(10)\right) ; 2.92\left(d d,{ }^{2} J(4 \mathrm{~b}, 4 \mathrm{a})=18.3\right.$, $\left.{ }^{3} J(4 \mathrm{~b}, 5)=9.4, \mathrm{H}_{\mathrm{b}}-\mathrm{C}(4)\right) ; 3.02-3.07(m, \mathrm{H}-\mathrm{C}(5)) ; 4.28-4.32(m, \mathrm{H}-\mathrm{C}(6)) ; 5.03\left(d,{ }^{3} J(1,5)=7.8\right.$, $\mathrm{H}-\mathrm{C}(1)) ; 7.31\left(d,{ }^{3} J\left(2^{\prime}, 3^{\prime}\right)=8.1,{ }^{3} J\left(6^{\prime}, 5^{\prime}\right)=8.1, \mathrm{H}-\mathrm{C}\left(2^{\prime}\right), \mathrm{H}-\mathrm{C}\left(6^{\prime}\right)\right) ; 7.58\left(d,{ }^{3} J\left(3^{\prime}, 2^{\prime}\right)=8.1,{ }^{3} J\left(5^{\prime}, 6^{\prime}\right)=8.1\right.$, $\left.\mathrm{H}-\mathrm{C}\left(3^{\prime}\right), \mathrm{H}-\mathrm{C}\left(5^{\prime}\right)\right) \cdot{ }^{13} \mathrm{C}-\mathrm{NMR}\left(\mathrm{CDCl}_{3}, 100 \mathrm{MHz}\right): 31.3(\mathrm{C}(10)) ; 32.6(\mathrm{C}(4)) ; 37.1(\mathrm{C}(9)) ; 40.3(\mathrm{C}(5))$; $76.7(\mathrm{C}(1)) ; 83.3(\mathrm{C}(6)) ; 125.8\left(q,{ }^{3} J(\mathrm{C}, \mathrm{F})=4.0, \mathrm{C}\left(3^{\prime}\right), \mathrm{C}\left(5^{\prime}\right)\right) ; 128.8\left(\mathrm{C}\left(2^{\prime}\right), \mathrm{C}\left(6^{\prime}\right)\right) ; 129.2\left(q,{ }^{2} J(\mathrm{C}, \mathrm{F})=\right.$ 32.1, $\left.\mathrm{C}\left(4^{\prime}\right)\right) ; 143.7\left(q,{ }^{5} J(\mathrm{C}, \mathrm{F})=1.3, \mathrm{C}\left(1^{\prime}\right)\right)$; $169.4(\mathrm{C}(8)) ; 173.1(\mathrm{C}(3))$. Anal. calc. for $\mathrm{C}_{15} \mathrm{H}_{13} \mathrm{~F}_{3} \mathrm{O}_{4}$ (314.26): C 57.33, H 4.17; found: C 57.26, H 4.43.

Preparation of 7a-7c. A soln. of magnesium methyl carbonate (MMC; 2.0M in DMF; 4.9, 5.2, and $4.7 \mathrm{ml}$, resp. $)$ was added to the bis- $\gamma$-lactone $7 \mathbf{a}(0.22 \mathrm{~g}, 0.89 \mathrm{mmol}), 7 \mathbf{b}(0.25 \mathrm{~g}, 0.96 \mathrm{mmol})$, or $7 \mathbf{c}(0.27 \mathrm{~g}$, $0.86 \mathrm{mmol}$ ) under $\mathrm{N}_{2}$. The mixture was stirred at $112^{\circ}$ for $5 \mathrm{~h}$ and then was poured into an ice-cold mixture of $6 \mathrm{M} \mathrm{HCl} / \mathrm{Et}_{2} \mathrm{O} 5: 1(24 \mathrm{ml})$, and stirred in order to dissolve the precipitate formed. The phases were separated, and extractions with $\mathrm{Et}_{2} \mathrm{O}$ were performed $(2 \times 10 \mathrm{ml})$. The combined org. phases were washed with sat. aq. $\mathrm{NaCl}$ soln. $(15 \mathrm{ml})$, dried $\left(\mathrm{Na}_{2} \mathrm{SO}_{4}\right)$, and concentrated i.v. To the yellow oil thus obtained, it was added a previously prepared mixture of $\mathrm{AcONa}(91,96$ and $87 \mathrm{mg}$ for $\mathbf{7 a}, \mathbf{7 b}$, and $\mathbf{7 c}$, resp. $)$, AcOH $(3.4 \mathrm{ml})$, formalin $(2.5 \mathrm{ml})$, and $\mathrm{Et}_{2} \mathrm{NH}(1.1 \mathrm{ml})$. The mixture was vigorously shaken for $1 \mathrm{~min}$ and then heated on a steam bath for $5 \mathrm{~min}$, cooled, and poured into $\mathrm{H}_{2} \mathrm{O}(27 \mathrm{ml})$ and $\mathrm{Et}_{2} \mathrm{O}(17 \mathrm{ml})$. The org. phase was washed with $\mathrm{H}_{2} \mathrm{O}(12 \mathrm{ml})$ and sat. aq. $\mathrm{NaHCO}_{3}$ soln. $(12 \mathrm{ml})$, and dried $\left(\mathrm{Na}_{2} \mathrm{SO}_{4}\right)$ and concentrated i.v. The white solid obtained was purified by $\mathrm{CC}\left(\mathrm{SiO}_{2}\right.$, hexane/AcOEt $1: 1$ to yield compounds 7a $(0,12 \mathrm{~g}, 53 \%)$, $7 \mathbf{b}(0,12 \mathrm{~g}, 45 \%)$, and $\mathbf{7 c}(0,10 \mathrm{~g}, 36 \%)$.

(1R,5R,6R)-4-Methylidene-6-(2-phenylethyl)-2,7-dioxabicyclo[3.3.0]octan-3,8-dione (7a). Colorless oil. $R_{\mathrm{f}}$ (hexane/AcOEt 1:1) 0.56. $[\alpha]_{\mathrm{D}}^{24}=+76.7\left(c=1.46, \mathrm{CH}_{2} \mathrm{Cl}_{2}\right)$. IR (film): 3061, 3027, 2925, 2856, 1789, 1665, 1603, 1497, 1455, 1297, 1221, 1105, 1064, 752, 702. ${ }^{1} \mathrm{H}-\mathrm{NMR}\left(\mathrm{CDCl}_{3}, 400 \mathrm{MHz}\right): 2.10-2.15(\mathrm{~m}$, $\left.\mathrm{CH}_{2}(9)\right) ; 2.75-2.92\left(m, \mathrm{CH}_{2}(10)\right) ; 3.54-3.58(m, \mathrm{H}-\mathrm{C}(5)) ; 4.41\left(d t,{ }^{3} J(6,5)=4.1,{ }^{3} J(6,9)=6.6, \mathrm{H}-\mathrm{C}(6)\right)$; $5.05\left(d,{ }^{3} J(1,5)=8.4, \mathrm{H}-\mathrm{C}(1)\right) ; 5.81\left(d,{ }^{2} J(11 \mathrm{a}, 11 \mathrm{~b})=2.2, \mathrm{H}_{\mathrm{b}}-\mathrm{C}(11)\right) ; 6.44\left(d,{ }^{2} J(11 \mathrm{~b}, 11 \mathrm{a})=2.2\right.$,

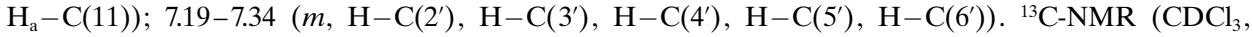
$100 \mathrm{MHz})$ : $31.3(\mathrm{C}(10)) ; 37.8(\mathrm{C}(9))$; $44.3(\mathrm{C}(5)) ; 74.1(\mathrm{C}(1)) ; 84.0(\mathrm{C}(6)) ; 126.4(\mathrm{C}(11)) ; 126.7\left(\mathrm{C}\left(4^{\prime}\right)\right)$; $128.3\left(\mathrm{C}\left(2^{\prime}\right), \mathrm{C}\left(6^{\prime}\right)\right) ; 128.8\left(\mathrm{C}\left(3^{\prime}\right), \mathrm{C}\left(5^{\prime}\right)\right) ; 134.3(\mathrm{C}(4)) ; 139.5\left(\mathrm{C}\left(1^{\prime}\right)\right) ; 167.3(\mathrm{C}(3)) ; 169.6(\mathrm{C}(8))$. Anal. calc. for $\mathrm{C}_{15} \mathrm{H}_{14} \mathrm{O}_{4}$ (258.27): C 69.76, H 5.46; found: C 69.87, H 5.66.

(1R,5R,6R)-4-Methylidene-6-[2-(4-methylphenyl)ethyl]-2,7-dioxabicyclo[3.3.0]octan-3,8-dione (7b). White solid. $R_{\mathrm{f}}$ (hexane/AcOEt 1:1) 0.57. M.p. 91.1-93.4 (hexane/AcOEt 1:1). $[\alpha]_{\mathrm{D}}^{21}=+68.7(c=1.15$, $\mathrm{CH}_{2} \mathrm{Cl}_{2}$ ). IR (KBr): 3044, 2923, 2862, 1791, 1760, 1663, 1515, 1297, 1240, 1128, 1038, 1019, 979, 818. ${ }^{1} \mathrm{H}-\mathrm{NMR}\left(\mathrm{CDCl}_{3}, 200 \mathrm{MHz}\right): 2.02-2.21\left(m, \mathrm{CH}_{2}(9)\right) ; 2.33(s, \mathrm{Me}) ; 2.67-2.88\left(m, \mathrm{CH}_{2}(10)\right) ; 3.53-3.60$ $(m, \mathrm{H}-\mathrm{C}(5))$; $4.32-4.45(m, \mathrm{H}-\mathrm{C}(6)) ; 5.06\left(d,{ }^{3} J(1,5)=8.5, \mathrm{H}-\mathrm{C}(1)\right) ; 5.81\left(d,{ }^{2} J(11 \mathrm{a}, 11 \mathrm{~b})=2.1\right.$, $\left.\mathrm{H}_{\mathrm{b}}-\mathrm{C}(11)\right) ; 6.43\left(d,{ }^{2} J(11 \mathrm{~b}, 11 \mathrm{a})=2.1, \mathrm{H}_{\mathrm{a}}-\mathrm{C}(11)\right) ; 7.06-7.15\left(m, \mathrm{H}-\mathrm{C}\left(2^{\prime}\right), \mathrm{H}-\mathrm{C}\left(3^{\prime}\right), \mathrm{H}-\mathrm{C}\left(5^{\prime}\right)\right.$, $\left.\mathrm{H}-\mathrm{C}\left(6^{\prime}\right)\right) .{ }^{13} \mathrm{C}-\mathrm{NMR}\left(\mathrm{CDCl}_{3}, 50 \mathrm{MHz}\right): 21.0(\mathrm{Me}) ; 30.8(\mathrm{C}(10)) ; 37.8(\mathrm{C}(9)) ; 44.2(\mathrm{C}(5)) ; 74.2(\mathrm{C}(1))$; $84.1(\mathrm{C}(6)) ; 126.4(\mathrm{C}(11)) ; 127.4\left(\mathrm{C}\left(3^{\prime}\right), \mathrm{C}\left(5^{\prime}\right)\right) ; 128.2\left(\mathrm{C}\left(2^{\prime}\right), \mathrm{C}\left(6^{\prime}\right)\right) ; 134.3(\mathrm{C}(4)) ; 136.2\left(\mathrm{C}\left(4^{\prime}\right)\right) ; 136.4$ 
$\left(\mathrm{C}\left(1^{\prime}\right)\right) ; 167.4(\mathrm{C}(3)) ; 169.7(\mathrm{C}(8))$. Anal. calc. for $\mathrm{C}_{16} \mathrm{H}_{16} \mathrm{O}_{4}(272.30)$ : $\mathrm{C} 70.57$, H 5.92; found: $\mathrm{C} 70.41, \mathrm{H}$ 5.85 .

(1R,5R,6R)-4-Methylidene-6-\{2-[4-(trifluoromethyl)phenyl]ethyl\}2,7-dioxabicyclo[3.3.0]octan-3,8dione (7c). Colorless oil. $R_{\mathrm{f}}$ (hexane/AcOEt 1:1) 0.61 . $[\alpha]_{\mathrm{D}}^{26}=+37.5\left(c=1.60, \mathrm{CH}_{2} \mathrm{Cl}_{2}\right)$. IR (film): 3118 , 2960, 2918, 2868, 1782, 1666, 1617, 1418, 1323, 1194, 1118, 1049, 1019, 846, 594. ${ }^{1} \mathrm{H}-\mathrm{NMR}\left(\mathrm{CDCl}_{3}\right.$, $400 \mathrm{MHz}): 2.10-2.19\left(m, \mathrm{CH}_{2}(9)\right) ; 2.80-2.88\left(m, \mathrm{H}_{\mathrm{a}}-\mathrm{C}(10)\right) ; 2.93-3.00\left(m, \mathrm{H}_{\mathrm{b}}-\mathrm{C}(10)\right) ; 3.56-3.60(m$, $\mathrm{H}-\mathrm{C}(5)) ; 4.35-4.42(m, \mathrm{H}-\mathrm{C}(6)) ; 5.08\left(d,{ }^{3} J(1,5)=6.4, \mathrm{H}-\mathrm{C}(1)\right) ; 5.85 \quad\left(d,{ }^{2} J(11 \mathrm{a}, 11 \mathrm{~b})=2.4\right.$, $\left.\mathrm{H}_{\mathrm{b}}-\mathrm{C}(11)\right) ; 6.46\left(d,{ }^{2} J(11 \mathrm{~b}, 11 \mathrm{a})=2.4, \mathrm{H}_{\mathrm{a}}-\mathrm{C}(11)\right) ; 7.33\left(d,{ }^{3} J\left(2^{\prime}, 3^{\prime}\right)=8.0,{ }^{3} J\left(6^{\prime}, 5^{\prime}\right)=8.0, \mathrm{H}-\mathrm{C}\left(2^{\prime}\right)\right.$, $\left.\mathrm{H}-\mathrm{C}\left(6^{\prime}\right)\right) ; 7.58\left(d,{ }^{3} J\left(3^{\prime}, 2^{\prime}\right)=8.0,{ }^{3} J\left(5^{\prime}, 6^{\prime}\right)=8.0, \mathrm{H}-\mathrm{C}\left(3^{\prime}\right), \mathrm{H}-\mathrm{C}\left(5^{\prime}\right)\right) .{ }^{13} \mathrm{C}-\mathrm{NMR}\left(\mathrm{CDCl}_{3}, 100 \mathrm{MHz}\right): 31.3$ $(\mathrm{C}(10)) ; 37.8(\mathrm{C}(9))$; $44.4(\mathrm{C}(5)) ; 74.1(\mathrm{C}(1)) ; 83.8(\mathrm{C}(6)) ; 125.8\left(q,{ }^{3} \mathrm{~J}(\mathrm{C}, \mathrm{F})=3.7, \mathrm{C}\left(3^{\prime}\right), \mathrm{C}\left(5^{\prime}\right)\right) ; 126.5$ $(\mathrm{C}(11)) ; 128.7\left(\mathrm{C}\left(2^{\prime}\right), \mathrm{C}\left(6^{\prime}\right)\right) ; 129.1\left(q,{ }^{2} J(\mathrm{C}, \mathrm{F})=32.7, \mathrm{C}\left(4^{\prime}\right)\right) ; 134.2(\mathrm{C}(4)) ; 143.7\left(\mathrm{C}\left(1^{\prime}\right)\right) ; 167.2(\mathrm{C}(3))$; $169.5(\mathrm{C}(8))$. Anal. calc. for $\mathrm{C}_{16} \mathrm{H}_{13} \mathrm{~F}_{3} \mathrm{O}_{4}$ (326.27): C 58.90, H 4.02; found: C 58.56, H 4.33.

Biological Assay. Four sterilized Blank paper disks $(6 \mathrm{~mm})$ were dipped into the solns. of the bis- $\gamma$ lactones $\mathbf{6 a - 6 c}, 7 \mathbf{a}-7 \mathbf{c}$, and avenaciolide (1000 ppm) in $\mathrm{CH}_{2} \mathrm{Cl}_{2}$. After $5 \mathrm{~min}$, the disks were allowed to dry in a desiccator at reduced pressure. The same procedure was employed in the preparation of the positive control (the commercial fungicide Folicur). The negative check treatment was prepared with solvent only. Each disk was placed in the center of a Petri dish containing Colletotrichum gloeosporioides spores (isolated from infected tissues of papaya) over the potato dextrose agar medium (DIFCO). The distances from the center of the disks to the edge of the inhibition zone, from where a dense and filamentous growth of the fungus could be observed with the aid of a stereoscopic microscope, were measured after $48 \mathrm{~h}$ at $25^{\circ}$. Compounds $6 \mathbf{a}-\mathbf{6 c}$ were inactive. The Petri dishes containing the bis- $\gamma$ lactones $7 \mathbf{a}-7 \mathbf{c}$ and avenaciolide showed transparent hales of $1.5,1.5,1.8$, and $1.7 \mathrm{~cm}$ diameter, resp., inside a larger hale containing some micelial growth. Tebuconazole (Folicur) presented an inhibition hale of $3.8 \mathrm{~cm}$

\section{REFERENCES}

[1] D. Brookes, B. K. Tidd, W. B. Turner, J. Chem. Soc. 1963, 68, 5385.

[2] R. C. Anderson, B. Fraser-Reid, J. Am. Chem. Soc. 1975, 97, 3870.

[3] D. L. Hughes, Acta Crystallogr., Sect. B 1978, 34, 3674.

[4] J. Meyer, P. M. Vignais, Biochim. Biophys. Acta 1973, 325, 375.

[5] D. Litwinska, A. Szczesna-Kaczmarek, J. Popinigis, Int. J. Biochem. 1984, 16, 943.

[6] V. S. Martín, C. M. Rodríguez, T. Martín, Org. Prep. Proced. Int. 1998, 30, 291.

[7] S. Braukmüller, R. Brückner, Eur. J. Org. Chem. 2006, 2110.

[8] A. S. Magaton, M. M. M. Rubinger, F. C. Macedo-Júnior, L. Zambolim, J. Braz. Chem. Soc. 2007, $18,284$.

[9] A. K. Picman, Biochem. Syst. Ecol. 1986, 14, 255.

[10] S. Czernecki, C. Georgoulouis, C. L. Stevens, K. Vijayakumaran, Tetrahedron Lett. 1985, 26, 1699.

[11] A. Rosenthal, L. Nguyen, J. Org. Chem. 1969, 34, 1029.

[12] W. L. Parker, F. Johnson, J. Org. Chem. 1973, 38, 2489.

[13] D. Brookes, S. Sternhell, B. K. Tidd, W. B. Turner, Aust. J. Chem. 1965, 18, 373.

[14] K. Lee, Y. Wu, I. H. Hall, J. Med. Chem. 1977, 20, 911.

[15] M. Miyazawa, H. Shimabayashi, S. Hayashi, S. Hashimoto, S. Nakamura, H. Kosaka, H. Kameoka, J. Agric. Food. Chem. 2000, 48, 5406.

[16] A. J. Scott, M. Knott, Biometrics 1974, 30, 507. 